\title{
Characterization and monitoring of GOES-16 ABI stray light and comparison with Himawari-8 AHI and GOES-17 ABI
}

\author{
Xi Shao $\odot,{ }^{a}, b, *$ Xiangqian Wu $\odot,{ }^{c}$ Fangfang $Y u,{ }^{a}$ and Changyong Cao \\ ${ }^{a}$ University of Maryland, Cooperative Institute for Satellite Earth System Studies, \\ Earth System Science Interdisciplinary Research Center, College Park, Maryland, \\ United States \\ ${ }^{b}$ University of Maryland, Department of Astronomy, College Park, Maryland, United States \\ ${ }^{c}$ NOAA National Environmental Satellite, Data, and Information Service, Center for \\ Satellite Applications and Research, College Park, Maryland, United States
}

\begin{abstract}
The Advanced Baseline Imager (ABI) aboard Geostationary Operational Environmental Satellite (GOES)-16 and -17 satellites represent the next-generation geostationary multispectral imaging instrument. Since GOES-16 ABI imagery data became available, stray light was observed in ABI visible, near-infrared (VNIR), and $3.9 \mu \mathrm{m}$, i.e., CH07, channels. A stray-light characterization scheme was developed to quantitatively monitor stray-light variation in ABI imagery. The stray-light analysis is focused on ABI CH07, whose nighttime radiometric performance being impacted by stray light is of main concern. It is found that the stray light in the ABI imagery occurs over $\sim 3$ months around spring and fall equinox each year. The maximum stray light of GOES-16 ABI CH07 is $\sim 0.65 \mathrm{~K}$ at $300 \mathrm{~K}$ scene in zone of normal performance (ZONP), i.e., region with relative solar angle $>7.5 \mathrm{deg}$, which is within the radiometric requirement of $1 \mathrm{~K}$. The analysis of Himawari-8 Advanced Himawari Imager (AHI) CH07 data indicates that its maximum stray light is $\sim 3.35 \mathrm{~K}$ at $300 \mathrm{~K}$ scene in ZONP, much higher than that of GOES-16. This confirms the effectiveness of reducing the major stray-light leaking path in ABI as a result of lessons learned from Himawari-8 AHI. The magnitude of GOES-17 ABI CH07 stray light is shown to be $\sim 0.45 \mathrm{~K}$ in ZONP, slightly lower than GOES-16. The analysis of AHI 3.9 and $6.2 \mu \mathrm{m}$ channel stray-light radiance ratio is shown to match the ratio of solar irradiance spectrum, which suggests that the stray-light issue is due to direct leakage of solar radiation through the instrument. Further characterization and monitoring of GOES-16 ABI VNIR channel stray light also help understand the solar origin of ABI stray light. This paper also investigated cases of strong atmospheric refraction-induced stray-light contamination onto ABI detectors during solar eclipse seasons and presents scheme to reduce such potentially harmful contamination. (C) The Authors. Published by SPIE under a Creative Commons Attribution 4.0 Unported License. Distribution or reproduction of this work in whole or in part requires full attribution of the original publication, including its DOI. [DOI: 10.1117/1.JRS.15.017503]
\end{abstract}

Keywords: Geostationary Operational Environmental Satellite-16; Geostationary Operational Environmental Satellite-17; Advanced Baseline Imager; stray light; Himawari-8 Advanced Himawari Imager; solar avoidance; refraction-induced stray light.

Paper 200834 received Nov. 24, 2020; accepted for publication Feb. 5, 2021; published online Feb. 26, 2021.

\section{Introduction}

The Geostationary Operational Environmental Satellite (GOES)-16 and -17 of the National Oceanic and Atmospheric Administration (NOAA) represent the next-generation satellite in geosynchronous orbit. The GOES-16 was launched on November 19, 2016, ${ }^{1-7}$ and was in operation initially at around $89.5^{\circ} \mathrm{W}$ longitude where it was undergoing an extended testing and validation phase and then moved to $75.2^{\circ} \mathrm{W}$ longitude as GOES-East on December 18, 2017, to cover the

*Address all correspondence to Xi Shao, xshao@umd.edu 
Eastern American continental regions. After the successful operation of GOES-16, GOES-17 was launched on March 1, 2018, ${ }^{8-10}$ and became operational as GOES-West at $137.2^{\circ} \mathrm{W}$ longitude on February 12, 2019.

The Advanced Baseline Imager (ABI) is the primary instrument aboard GOES-16 and -17 and represents the next-generation visible, near-infrared (VNIR), and infrared (IR) imager at geosynchronous orbit. It has 16 multispectral channels, including six VNIR and 10 thermal emissive bands. The new design elements of the ABI including double-scan mirrors, onboard solar diffuser, and controlled calibration target temperature improve its radiometric accuracy. Two scanning mirrors of ABI control the East/West (E/W) and North/South (N/S) scans to produce the full-disk (FD) ABI imagery. The ABI is capable of generating FD imagery every 5, 10, and $15 \mathrm{~min}$. The spatial resolution varies from 0.5 to $2 \mathrm{~km}$ for different spectral channels. With its significantly improved spectral, spatial and temporal resolution and radiometric quality, the ABI continues to provide valuable data for climate and weather research studies and greatly improve the capability of weather forecasting and environmental monitoring.

Before the launch of GOES-16 ABI, the Advanced Himawari Imager (AHI) onboard Himawari-8 satellite of Japan Meteorological Agency was launched on October 7, 2014, ${ }^{11-19}$ and was in operation at $140.7^{\circ}$ East longitude covering the East Asia and Western Pacific regions. The AHI instrument was built by the same vendor as the ABI onboard the GOES-16 and -17 satellite. Both AHI and ABI have very similar optical design. The $\sim 2$ years lead time of AHI provided invaluable opportunity to prepare for a successful ABI mission through early access and analysis of AHI data. Shortly after Himawari-8 AHI data became available, it was found in February 2015 that there were significant stray-light contamination in VNIR channel and IR channel ( $\mathrm{CH} 07$ and $\mathrm{CH} 08)$ of $\mathrm{AH} .{ }^{18,19}$ Root cause was later identified as the contamination of AHI sensor around solar eclipse season by the leaking of solar light into the instrument. As the result of lessons learned from analysis of Himawari- 8 AHI data, the optical path of major stray-light leaking in AHI was identified by the instrument vendor and fixes were applied to GOES-16 and -17 ABI to remedy the stray light.

After GOES-16 ABI data became available, characterization and monitoring of stray light in $\mathrm{ABI}$ imagery have been routinely performed. It was found that there were remnant stray light in the VNIR and CH07 imagery of ABI around solar eclipse season. In this paper, we developed a stray-light characterization scheme to quantitatively monitor the occurrence, location, and magnitude of stray-light variation in ABI FD imagery. Since the impacts of stray light on the nighttime radiometric performance of ABI $3.9 \mu$ m channel, i.e., $\mathrm{CH} 07$, are of major concern, the stray-light monitoring is focused on this mid-wave IR (MWIR) channel. The GOES-16 CH07 stray-light pattern and magnitude are also compared with that of Himawari-8 AHI to evaluate the effectiveness of ABI design modifications in reducing the strong stray-light source observed in AHI. The consistency of stray-light magnitude and variation between GOES-16 and -17 ABI are also evaluated.

The stray-light pattern and variation in GOES-16 ABI VNIR (CH01-06) channels are also reported in this paper. Although there are no radiometric requirements for ABI VNIR channel data during night, such analysis of ABI multi-channel data helps understand the solar origin of ABI stray light. There were cases of strong atmospheric refraction-induced stray-light contamination onto ABI detectors during solar eclipse season which can be harmful to these detectors. We analyzed the solar avoidance parameter setting during these events and demonstrated schemes to avoid or reduce such potentially harmful strong stray-light contamination.

This paper is organized as following. In Sec. 2, we first present typical features of the GOES-16 ABI CH07 stray light. Then, we present a stray-light characterization scheme based on time-difference processing and Sun-centered coordinate (SCC) transformation of ABI imagery data. The scheme is applied to perform long term monitoring of GOES-16 ABI CH07 stray light. Comparisons of $\mathrm{CH} 07$ stray light among GOES-16 and -17 ABI and Himawari-8 AHI are presented in Sec. 2.4. In Sec. 3, characterization and monitoring of GOES-16 ABI VNIR channel stray light are presented. In Sec. 4, the events of atmospheric refraction-induced strong stray-light contamination onto GOES-16 ABI detectors are analyzed. The remediation scheme to avoid or reduce such potentially harmful stray-light contamination is also reported. 


\section{Characterization and Monitoring of $\mathrm{ABI} \mathrm{CH} 07$ Stray Light}

\subsection{Typical Stray-Light Pattern of $\mathrm{ABI} C \mathrm{CHO7}$}

The seventh band (CH07) of ABI is an MWIR channel with center wavelength at $3.9 \mu \mathrm{m}$ and has been designed to monitor temperature variation of low cloud, fire and fog. The spatial resolution for this band is $2 \mathrm{~km}$. The radiometric calibration of ABI IR channels is performed with the measurements of the internal calibration target of blackbody and deep space. In Ref. 7, brightness temperature biases for GOES-16 ABI IR bands were assessed to meet radiometric specification by cross-comparison between the measurements of hyperspectral IR sounder, i.e., cross-track infrared sounder, on S-NPP/NOOAA-20 and GOES-16 ABI.

The stray light in the ABI CH07 FD imagery of Earth are mostly observed over $\sim 3$ months during February to April around spring equinox and October to November around fall equinox each year. Figure 1 shows typical location of Sun relative to Earth in the ABI fixed grid coordinate (FGC) when the stray light appeared at the southern edge of ABI imagery on February 13, 2017. The ABI- FGC is defined as on a plane perpendicular to the ABI-to-nadir direction. The $x$ and $y$ axis of the ABI-FGC is the E/W and N/S angle relative to ABI nadir, respectively. As shown in Fig. 1, Sun is located at $\sim 13.11^{\circ} \mathrm{S}$ of the ABI nadir. Due to the Earth's rotation during the scan, Sun moved from west-to-east relative to Earth during UT 5:30 to 7:00 on February 13, 2017. This is a typical geometric configuration among Sun-ABI-Earth when stray light appears. In this configuration, Sun and GOES-16 are located at the opposite side to Earth and GOES-16 ABI observes the night side of Earth. Since Sun is below the southern edge of Earth, solar light has direct path to illuminate onto the ABI instrument even though the ABI observes nighttime Earth.

The stray light appears as additional radiance superimposed onto the Earth scene image. The magnitude of the stray light is typically larger than the change of Earth scene radiance in sequential images. To quantitatively assess and monitor the effects of stray light on ABI imagery products, frame-to-frame difference images between consecutive ABI imagery were derived. As an example, Fig. 2 shows consecutive ABI Mode-4 (5-min apart) CH07 observations in radiance together with the time-difference image [Fig. 2(c)]. The boundaries at $5 \mathrm{deg}$ (red line) and $7.5 \mathrm{deg}$ (purple dash line) around Sun have been overlaid onto the ABI time-difference image in Fig. 2(c). The blue and yellow spikes at the southern bottom of Fig. 2(c) show the stray light in the previous and current image of ABI, respectively.

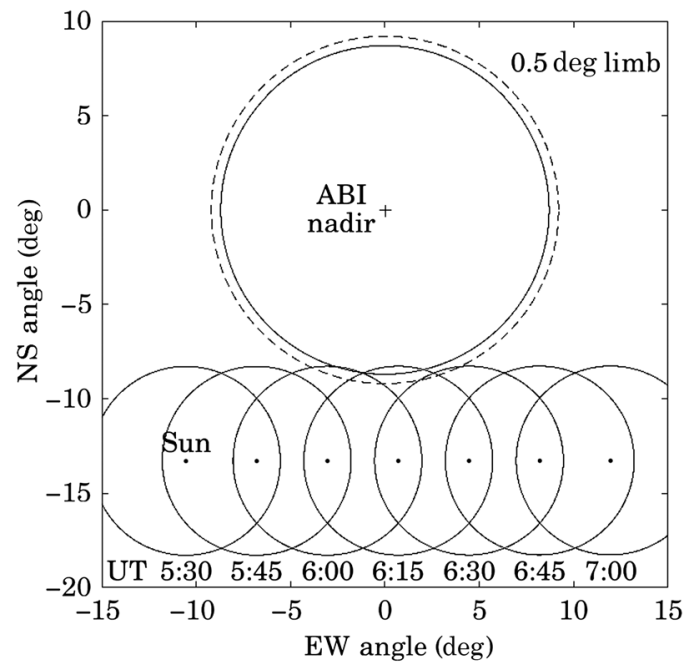

(a)

Fig. 1 (a) Relative position of Sun to Earth from UT 5:30 to 7:00 on February 13, 2017, projected onto the plane perpendicular to the direction of the ABI-to-nadir, i.e., in the ABI-FGC. The $x$ and $y$ axis are the $E / W$ and $N / S$ angle relative to the $A B I$ nadir, respectively. The solar position is to the south side of Earth and marked every 15 min with a 5-deg circle around the Sun, which appears to move from west to east. The nominal sub-satellite longitude is $89.5^{\circ} \mathrm{W}$. 


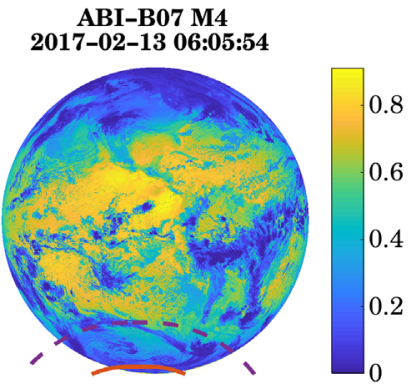

(a)

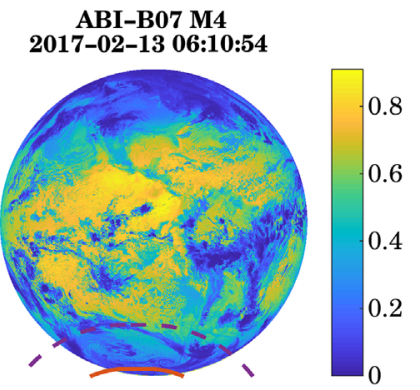

(b)

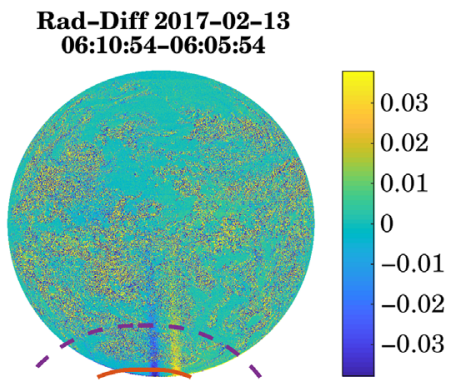

(c)

Fig. 2 (a) and (b) Example of consecutive GOES-16 ABI CH07 Mode-4 (5-min apart) observations in radiance $\left(\mathrm{mW} / \mathrm{m}^{2} \mathrm{srcm}^{-1}\right)$ on February 13, 2017. (c) Stray light is identifiable in the timedifference radiance $\left(\mathrm{mW} / \mathrm{m}^{2} \mathrm{srcm}^{-1}\right)$ image with the overlaying zone boundaries at 5 deg (red solid line) and $7.5 \mathrm{deg}$ (purple dash line) around the Sun. The region between the dash and solid lines of the $A B I F D$ imagery is marked as ZORP and the region outside the purple dash line in the FD imagery is referred as ZONP.

\subsection{Quantitative Characterization of $\mathrm{ABI} C H 07$ Stray Light}

Due to the stray-light contamination onto ABI imagery, which directly affects their radiometric performance, the ABI radiance images are divided into several zones with different radiometric performance requirements. The zone boundaries are defined in term of angular radius around Sun $\left(R_{\text {pixel-Sun }}\right)$ in ABI-FGC. Table 1 summarizes the zone boundaries for different ABI channels. The exclusion zone, aka solar avoidance zone, is defined as the interception area of ABI FD image with the projected circular area having angular radius $R_{1}$ around Sun. In addition, Sun is required to be outside of the 8.1-deg annulus circle around the ABI nadir. In this zone, the swaths of each ABI channel are truncated for pixels with $R_{\text {pixel-Sun }}<\sim 3$ deg to avoid direct illumination of solar light onto the detectors. For ABI VNIR channel CH01 to $\mathrm{CH} 06$ and IR channel $\mathrm{CH} 08$ to $\mathrm{CH} 16, R_{1}$ is set to be $3 \mathrm{deg}$. For $\mathrm{ABI} \mathrm{CH} 07, R_{1}$ is relaxed to be $5 \mathrm{deg}$ to account for much stronger stray light impacting this channel in comparison with other IR channels. In this exclusion zone, there is no requirement on the radiometric quality of imagery products. There is also a so-called zone of reduced performance (ZORP), which is located within the ring that is formed by $R_{1}<R_{\text {pixel-Sun }}<R_{2}$ and intercepts with ABI swaths. $R_{2}$ is set as fixed at 7.5 deg for all $\mathrm{ABI}$ channels. The regions in ABI imagery with $R_{\text {pixel-Sun }}>R_{2}$ or when Sun is fully behind Earth, i.e., Sun is inside the 8.1-deg annulus circle around ABI nadir, are called zone of normal performance (ZONP). For ABI VNIR channels, there are no radiometric requirements for nighttime observation so that the zone boundaries for these channels are set just for performance monitoring. For ABI IR channels ( $\mathrm{CH} 07$ to $\mathrm{CH} 16)$, the radiometric uncertainties are required to be within $1 \mathrm{~K}$ and $2 \mathrm{~K}$ at $300 \mathrm{~K}$ scene temperature for imagery data in ZONP and ZORP,

Table 1 Definition of different zones in terms of angular radius around the Sun $\left(R_{\text {pixel-Sun }}\right)$ and associated radiometric uncertainty requirements for $A B I$ VNIR and IR channels. The solar position and zone radius have both been projected onto $A B I-F G C$.

\begin{tabular}{lccc}
\hline \hline & Exclusion zone & ZORP & ZONP \\
\hline $\begin{array}{l}\text { ABI CH01-06 (VNIR) } \\
\text { zone definition }\end{array}$ & $R_{\text {pixel-Sun }} \leq 3 \mathrm{deg}$ & $3 \mathrm{deg}<R_{\text {pixel-Sun }} \leq 7.5 \mathrm{deg}$ & $R_{\text {pixel-Sun }}>7.5 \mathrm{deg}$ \\
$\mathrm{ABI}$ CH07 zone definition & $R_{\text {pixel-Sun }} \leq 5 \mathrm{deg}$ & $5 \mathrm{deg}<R_{\text {pixel-Sun }} \leq 7.5 \mathrm{deg}$ & $R_{\text {pixel-Sun }}>7.5 \mathrm{deg}$ \\
$\mathrm{ABI}$ CH08-16 zone definition & $R_{\text {pixel-Sun }} \leq 3 \mathrm{deg}$ & $3 \mathrm{deg}<R_{\text {pixel-Sun }} \leq 7.5 \mathrm{deg}$ & $R_{\text {pixel-Sun }}>7.5 \mathrm{deg}$ \\
$\begin{array}{l}\mathrm{ABI} \mathrm{CH} \text { 01-06 (VNIR) } \\
\text { uncertainty requirement }\end{array}$ & N.A. & $\begin{array}{c}6 \% \text { of full albedo over } \\
\text { day time scene }\end{array}$ & $\begin{array}{c}3 \% \text { of full albedo over } \\
\text { day time scene }\end{array}$ \\
$\begin{array}{l}\text { ABI CH07-16 uncertainty } \\
\text { requirement }\end{array}$ & N.A. & $2 \mathrm{~K}$ at 300 K scene & $1 \mathrm{~K}$ at 300 K scene \\
\hline \hline
\end{tabular}


respectively. Figure 2 shows examples of exclusion zone (within red ring), ZORP (between red and purple ring), and ZONP (outside of purple ring) relative to ABI CH07 Mode-4 (5-min apart) FD images and time-difference radiance image on February 13, 2017. The zone boundaries account for Sun's motion relative to Earth over the 5-min Mode-4 data acquisition time.

Analysis algorithms have been developed to process $\mathrm{ABI}$ imagery data for routine monitoring of stray-light magnitude in ZORP and ZONP for ABI VNIR and IR bands. In this section, we focus on using the $\mathrm{ABI} \mathrm{CH} 07$ as an example to illustrate the methodology and steps involved in quantitatively characterizing and monitoring the stray light within ZORP and ZONP. It is also noted that stray light in ABI IR channels with wavelength higher than $\mathrm{CH} 07$ are lower than the noise level. Therefore, the focus of this section is on the characterization and long term monitoring of ABI CH07 stray light.

\subsubsection{Time-difference processing and coordinate transformation}

The first step in stray-light analysis for ABI IR channel is to perform time-difference processing between sequential FD images as shown in Fig. 2 to remove the substantial background IR emissions at night. This can be readily done since ABI uses fixed grid to record data. Since the amplitude and feature size of these stray light are typically $>5$ to 15 -min change of Earth scene radiance, the derived time-difference image reveals ABI stray light more clearly. Figure 3(a) shows the four selected ABI CH07 time-difference images from UT 5:40 to UT 6:20 on February 21, 2017. These ABI Mode-4 FD data are recorded at regular 5-min intervals. The chosen images at irregular time instants were aimed to show the stray-light motion being aligned with Sun's position and the truncations of the ABI swaths for solar avoidance when Sun is at different $\mathrm{E} / \mathrm{W}$ positions. The blueish spike at the southern bottom of the time-difference image is due to stray light in the previous $\mathrm{ABI} \mathrm{CH} 07$ image that has been subtracted. The reddish spike to the East is due to stray light in the more recent ABI FD image. The stray light spike at the southern edge moves across the ABI FD image from west-to-east over about $1 \mathrm{~h}$. This corresponds to $\sim 15$ deg movement of Sun along the west-to-east direction due to Earth's rotation.
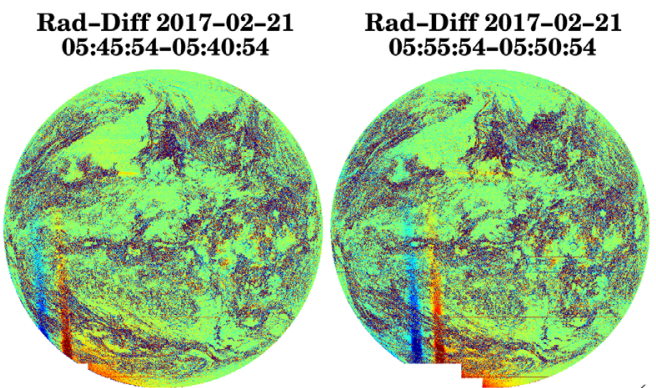

(a)
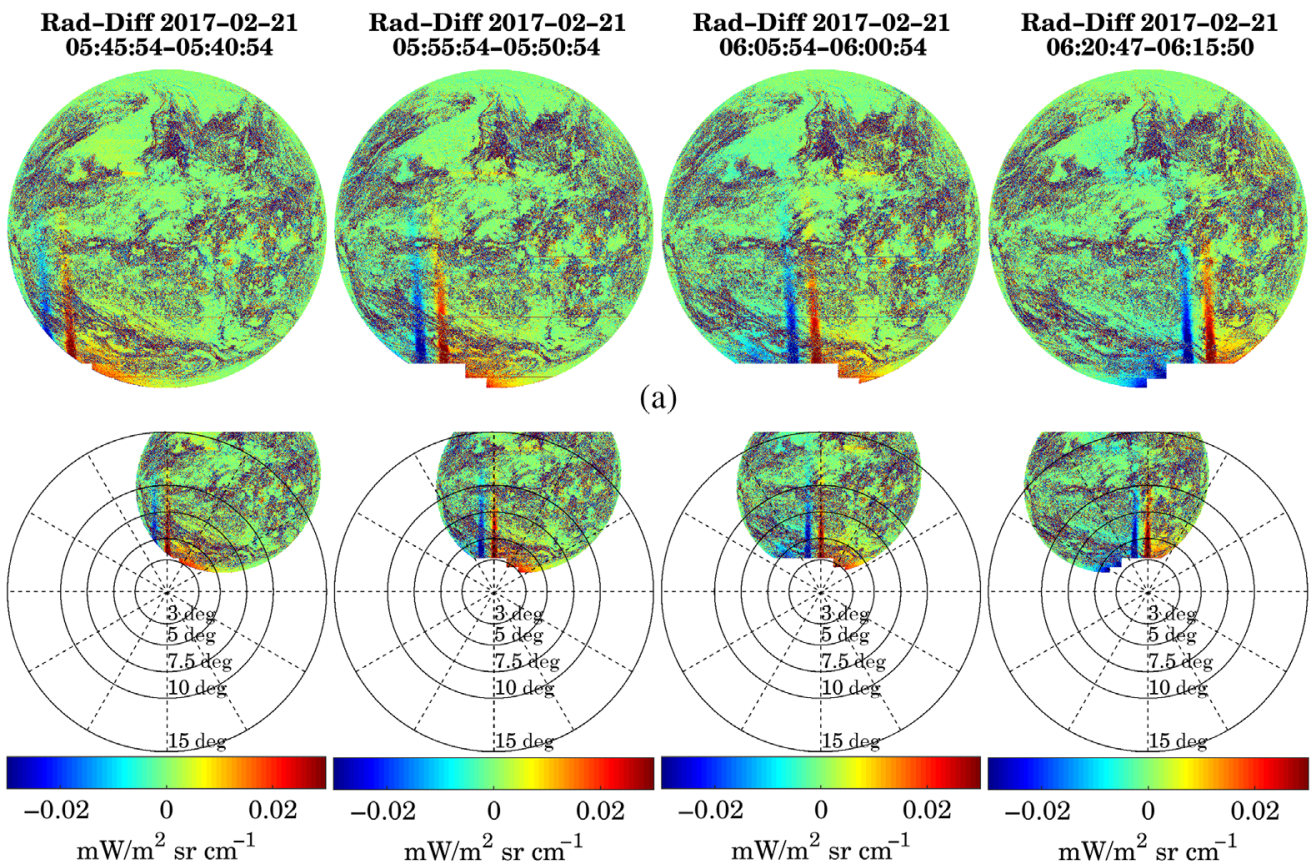

(b)

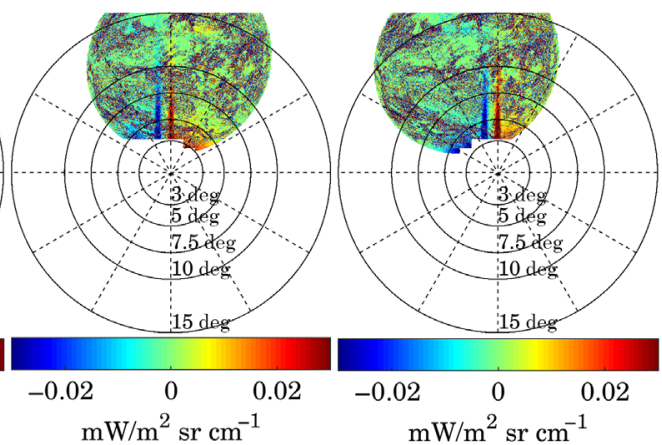

Fig. 3 (a) Selected GOES-16 ABI Mode-4 CH07 time-difference of radiance $\left(\mathrm{mW} / \mathrm{m}^{2}-\mathrm{sr}^{-\mathrm{cm}^{-1}}\right)$ images from UT 5:40 to UT 6:20 on February 21, 2017. (b) Illustration of invariant location of $\mathrm{ABI} \mathrm{CHO7}$ stray light spike at the bottom of FD image in SCC. The color scales are all of radiance unit $\mathrm{mW} / \mathrm{m}^{2} \mathrm{sr} \mathrm{cm}^{-1}$. The label in the top row indicates the time of FD images used to generate the radiance-difference image shown in each column. 
The swath-truncation due to solar avoidance in the exclusion zone can also be identified in the difference images, which moved along with Sun.

After deriving the time-difference image between sequential FD images in the ABI-FGC, the coordinate transformation to convert the $\mathrm{ABI}$ data from $\mathrm{FGC}$ to $\mathrm{SCC}$ was performed. The coordinate transformation was performed to convert the FGC of each ABI swath to SCC using the corresponding solar position during the swath at current scan so that the relative solar motion was removed and the data of each ABI swath were sun-centered. Examples of the ABI CH07 imagery data after coordinate transformation are shown in the bottom row of Fig. 3. The center of SCC is the position of Sun and circles mark the rings of $3 \mathrm{deg}, 5 \mathrm{deg}, 7.5 \mathrm{deg}, 10 \mathrm{deg}$, and $15 \mathrm{deg}$ radius around Sun. It can be seen that the position of stray light, i.e., the red spike, is fixed along the vertical axis in SCC, which is mainly due to the use of solar position aligned with the current ABI FD scan in the coordinate transformation. This demonstrates the stray light's solar origin and its co-movement with Sun. This coordinate transformation also makes the ZORP boundaries at $5 \mathrm{deg}$ and $7.5 \mathrm{deg}$ become concentric rings around Sun, which makes the monitoring of stray light inside ZORP and ZONP much easier.

\subsubsection{Estimation of stray light magnitude}

To monitor the ABI stray light, we need to be able to identify the stray-light-contaminated region, record the magnitude and location of the peak stray light inside ZORP and ZONP. As observed in SCC, when the Sun is at the southern or northern side of Earth, the peak stray light penetrates into the FD image along the vertical axis, i.e., N/S direction. When the Sun is near the equator, the stray light penetrates into the FD image along the horizontal axis, i.e., E/W direction. Figure 4 shows the example of data processing steps to derive stray-light amplitude and uncertainty in ZORP and ZONP. In our analysis, the time-difference data along the peak stray-light path (N/S direction) in SCC were first averaged perpendicular to and along the peak path. The resulting averaged stray light data is shown as the blue curve in Fig. 4 which starts at 3 deg with respect to the Sun. As the N/S angle increases, the stray light decreases as it is further away from the Sun and penetrates into the ABI FD. To quantitatively characterize the stray-light amplitude, the noise or fluctuation due to the time-varying background need to be removed. In our analysis, an exponential fitting of E/W-averaged time-difference data as a function of N/S angle in SCC has been applied. In Fig. 4, the solid red line is the fitted curve and dashed

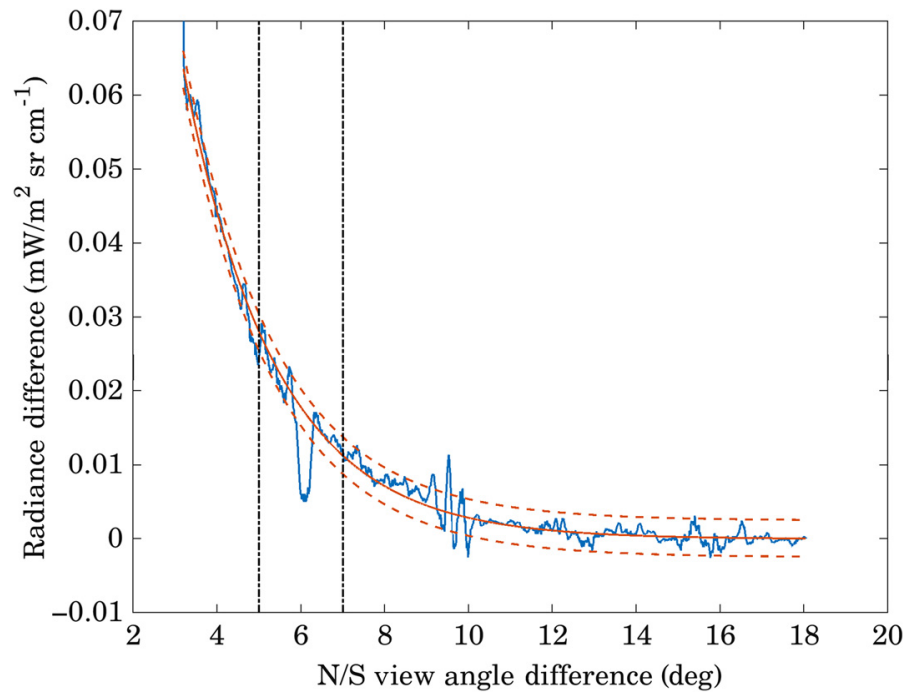

Fig. 4 Example of estimating stray light inside ABI ZORP and ZONP with exponential fitting. The averaged time-difference data in this figure are derived from the stray-light data shown in the second column of Fig. 3. Vertical lines mark the $5 \mathrm{deg}$ and $7.5 \mathrm{deg}$ boundary of ZORP and ZONP for ABI B07. Fitted line (red solid line) and envelope (red dashed lines) show the estimated stray-light magnitude and uncertainty, respectively, versus N/S angle in SCC. 
Shao et al.: Characterization and monitoring of GOES-16 ABI stray light and comparison...

Table 2 GOES-16 ABI scan modes during stray-light monitoring.

\begin{tabular}{lcc}
\hline \hline ABI scan mode \# & Scan duration (min) & Time interval \\
\hline 4 & 5 & January 28 to February 21, 2017 (PLT) \\
3 & 15 & February 22, 2017 to April 1, 2019 \\
6 & 10 & After April 2, 2019 \\
\hline \hline
\end{tabular}

Table 3 GOES-16 ABI nadir location change and stray-light occurrence interval.

\begin{tabular}{lccc}
\hline \hline Time interval & $\begin{array}{c}\text { ABI nadir location } \\
\text { (longitude, latitude) }\end{array}$ & $\begin{array}{c}\text { Yearly stray-light } \\
\text { occurrence interval }\end{array}$ & $\begin{array}{c}\text { Daily stray-light } \\
\text { watch out interval }\end{array}$ \\
\hline Launch to November 30, 2017 & $(-89.5,0)$ & $\begin{array}{c}\text { February to April, 2017 and } \\
\text { August to October, 2017 }\end{array}$ & $\sim$ UT 5:15 to UT 6:45 \\
After December 18, 2017 & $(-75.2,0)$ & $\begin{array}{c}\text { February to April and } \\
\text { August to October each year }\end{array}$ & UT 4:15 to UT 5:45 \\
\hline \hline
\end{tabular}

lines indicate the uncertainty in the stray-light amplitude estimation. The peak stray-light amplitude inside ZORP and ZONP are therefore marked as the absolute values of the fitted data at $\mathrm{N} / \mathrm{S}$ angle $=5 \mathrm{deg}$ and $7.5 \mathrm{deg}$ in SCC, respectively.

\subsection{Trending of GOES-16 ABI CHO7 Stray Light}

The GOES-16 ABI stray light in ZORP and ZONP were routinely monitored since the January 28,2017 . Monitoring of ABI stray light are performed with ABI FD imagery $1 \mathrm{~h}$ before and after satellite midnight throughout the year with the focus placed on the $\sim 50$ days before and after the equinox. There were several FD scan modes with different durations being in use for GOES-16 ABI. Table 2 summaries the scan modes and their associated scan duration and operational interval that were analyzed for GOES-16 ABI stray-light monitoring. Mode 4 was used during postlaunch testing (PLT) for ABI stray-light characterization. As part of special scan for stray-light test during PLT, the ABI Mode-4 FD data were routinely acquired at 5-min resolution around midnight whenever the opportunity for the special scan is available during this period. After the PLT for GOES-16 ABI stray-light test finished, Mode 3 with 15-min FD scan duration was in operation until April 2, 2019 when the scan mode was switched to Mode 6 with 10-min FD scan duration. In addition, there was the migration of GOES-16 ABI nadir from $-89.5^{\circ}$ to $-75.2^{\circ} \mathrm{W}$ longitude during early December of 2017 and GOES-16 became GOES-East on December 18, 2017. Corresponding to the change of GOES-16 ABI nadir, the daily interval of stray light occurrence also changed as shown in Table 3. The analysis of GOES-16 ABI FD radiance data for stray-light monitoring have taken into account of both the ABI scan mode change and the ABI nadir change during its lifetime.

\subsubsection{Daily evolution of GOES-16 ABI CHO7 stray light}

Typical examples of ABI CH07 stray-light appearing in the southern, near equator and northern part of ABI FD image are shown in Fig. 5-7, respectively.

After processing the ABI FD data with the stray-light analysis algorithm outlined in Sec. 2.2, the daily trends of maximum stray-light magnitude $\left(\mathrm{mW} / \mathrm{m}^{2} \mathrm{srcm}^{-1}\right)$ in ZORP and ZONP are shown in Fig. 5-7. In general, the stray-light moves along with the Sun in the E/W direction around local midnight and in the N/S direction over eclipse seasons. When the Sun is at the southern or northern side of Earth as shown in Figs. 5 and 7, respectively, the peak stray light always appears along the N/S axis in SCC and its location moves eastward over several FD images around the midnight. When the Sun is near the Earth's equator as shown in Fig. 6, the stray light appears at the western and eastern side of Earth when Sun enters and exits the FD 


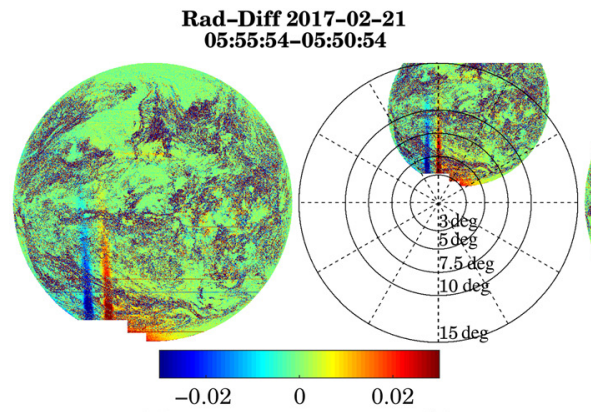

(a) (b)

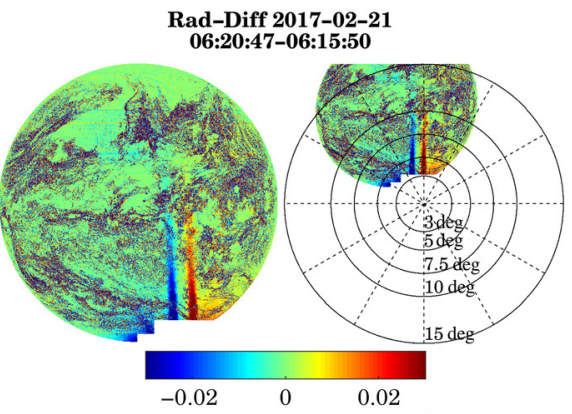

(c) (d)

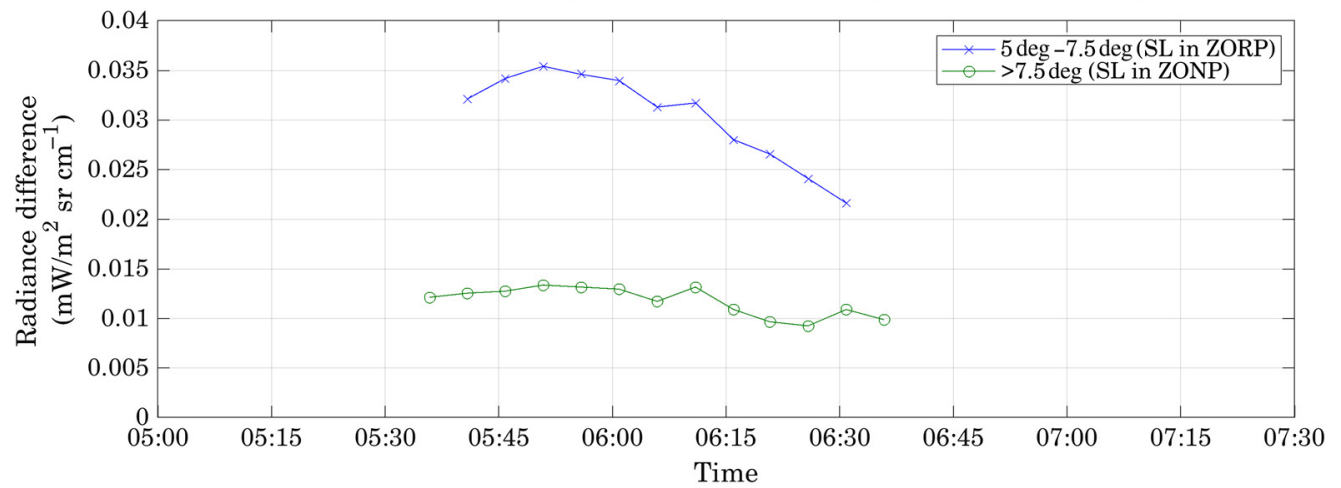

(e)

Fig. 5 (a)-(d) Typical stray-light pattern at the southern part of the ABI CH07 radiance-difference $\left(\mathrm{mW} / \mathrm{m}^{2} \mathrm{srcm}^{-1}\right)$ image. Both images in ABI FGC and SCC coordinates at two instants are shown, illustrating the appearance of stray light at fixed location in SCC. This set of ABI FD data was collected under Mode 4 with 5-min FD scan interval. (e) The daily evolution of $\mathrm{ABI} \mathrm{CH} 07$ straylight radiance $\left(\mathrm{mW} / \mathrm{m}^{2} \mathrm{srcm}^{-1}\right)$ on February 21, 2017. Blue and green lines are the maximum stray-light magnitude inside ZORP and ZONP, respectively.

imagery. There is no stray light when the Sun is behind Earth. We note that the FD data in Fig. 7 were recorded under ABI Mode-3 which was of 15-min FD-scan interval while Fig. 3 data were recorded using ABI Mode-4 FD scan which was specially tasked for stray-light characterization and was of 5-min interval. The different FD scan time interval (15 min versus 5 min) makes the horizontal distance between the blue and red stray-light spikes in Fig. 7 wider than that in Fig. 3.

\subsubsection{Long term trending of GOES-16 ABI CHO7 stray light}

The daily maximum magntiude of ABI CH07 stray light in ZORP and ZONP are recorded to assemble time series for long term monitoring. Figure 8(a) shows the long term trending of ABI $\mathrm{CH} 07$ stray light in ZORP. The E/W and N/S angular locations of the daily maximum stray light in ZORP togetehr with the corresponding solar position in ABI-FGC are shown in the second and third panel of Fig. 8(a). In Fig. 8(b), the trended daily maximum stray-light amplitude in ZONP is shown together with the E/W and N/S angular locations of the daily maximum stray light in ZONP.

Over the 3 month interval around spring and fall equinox each year, the daily peak stray light in both ZONP and ZORP have similar time-varition pattern with different amplitude. The contamination of stray light on ABI FD imagery occurs when the solar declination angle, i.e., the angular position of the Sun along the N/S direction in FGC, varies from $-17 \mathrm{deg}$ to $17 \mathrm{deg}$ as the Sun transits from south-to-north during February to early May and from $17 \mathrm{deg}$ to $-17 \mathrm{deg}$ as the Sun transits from north-to-south during August to early November each year. The timeevolution of daily peak stray light in both ZORP and ZONP consists of several phases such as the initial amplitude-increase phase when the solar delcination angle enters the $\sim 17$ - deg zone above or below Earth's equator; reaching-peak when the Sun transits at the edge of Earth's FD; sharp dipping, fltatterning and sharp increase in amplitude when the Sun transits across the FD of 
Shao et al.: Characterization and monitoring of GOES-16 ABI stray light and comparison...

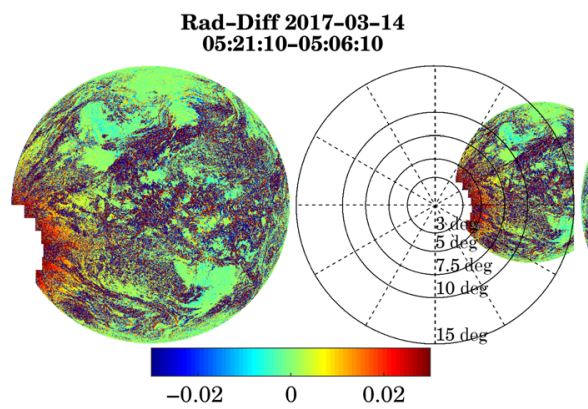

(a) (b)

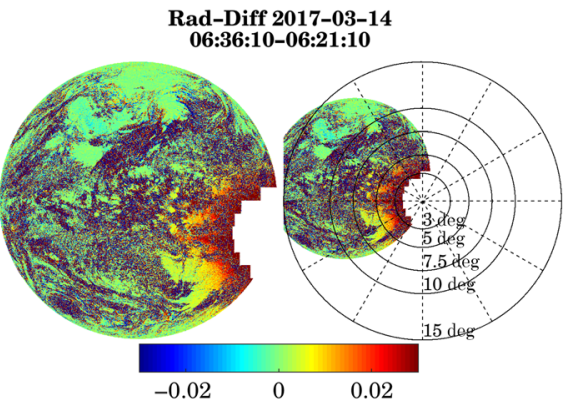

(c) (d)

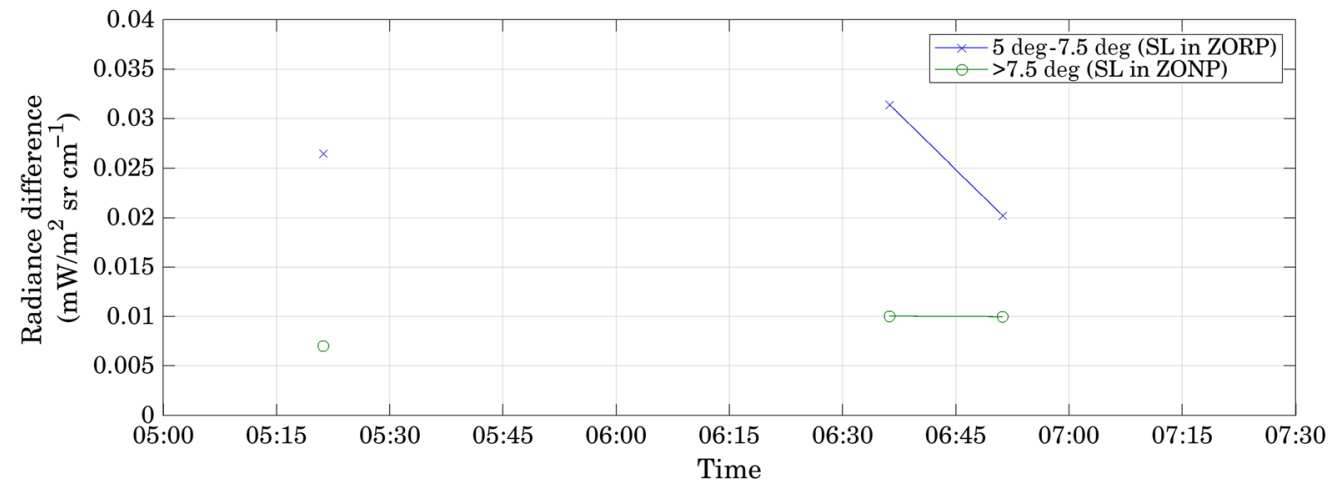

(e)

Fig. 6 Panels (a)-(e) arranged in the same format as in Fig. 5 to illustrate typical stray-light pattern and daily trend when the Sun transits near the Earth's equator on March 14, 2017. This set of ABI data was collected under Mode 3 with 15-min FD scan interval.

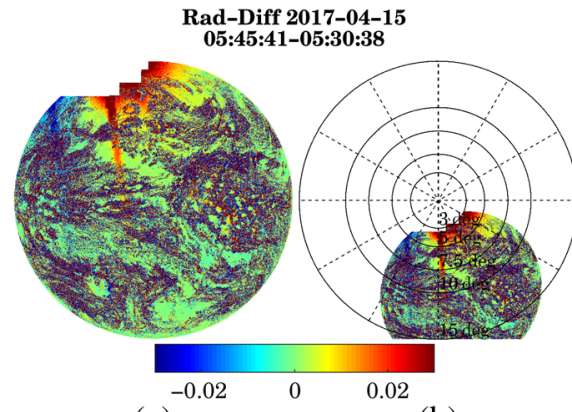

(a) (b)

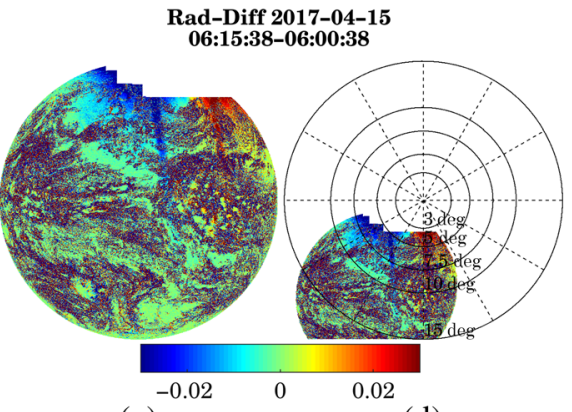

(c) (d)

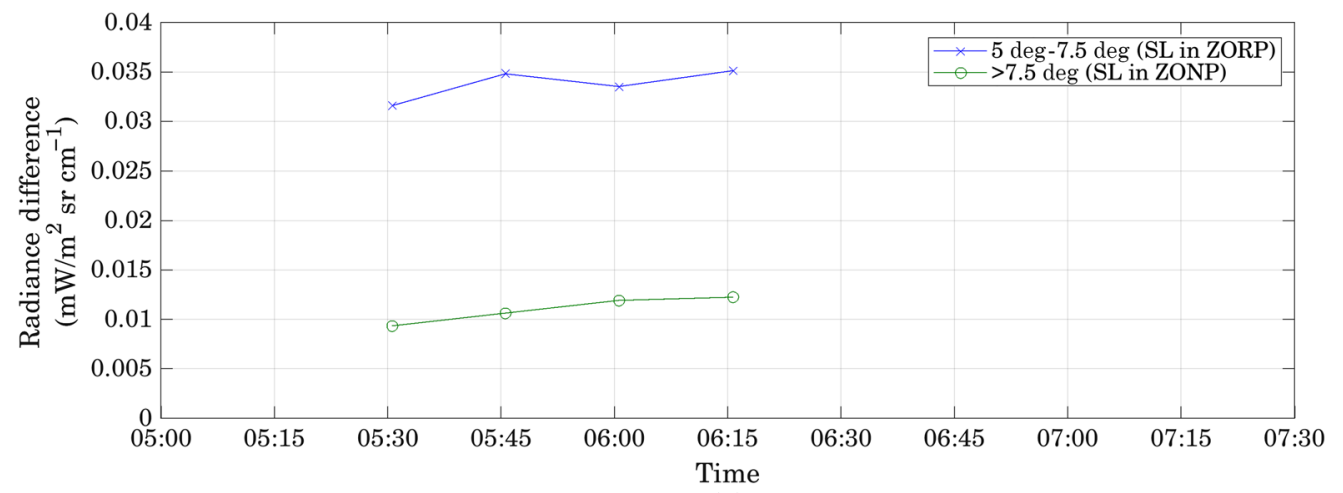

(e)

Fig. 7 Panels (a)-(e) arranged in the same format as in Fig. 5 to illustrate typical stray-light pattern and daily trend when the Sun transits above the Earth's northern pole on April 15, 2017. The ABI data was collected under Mode 3 with 15-min FD scan interval. 
Shao et al.: Characterization and monitoring of GOES-16 ABI stray light and comparison...
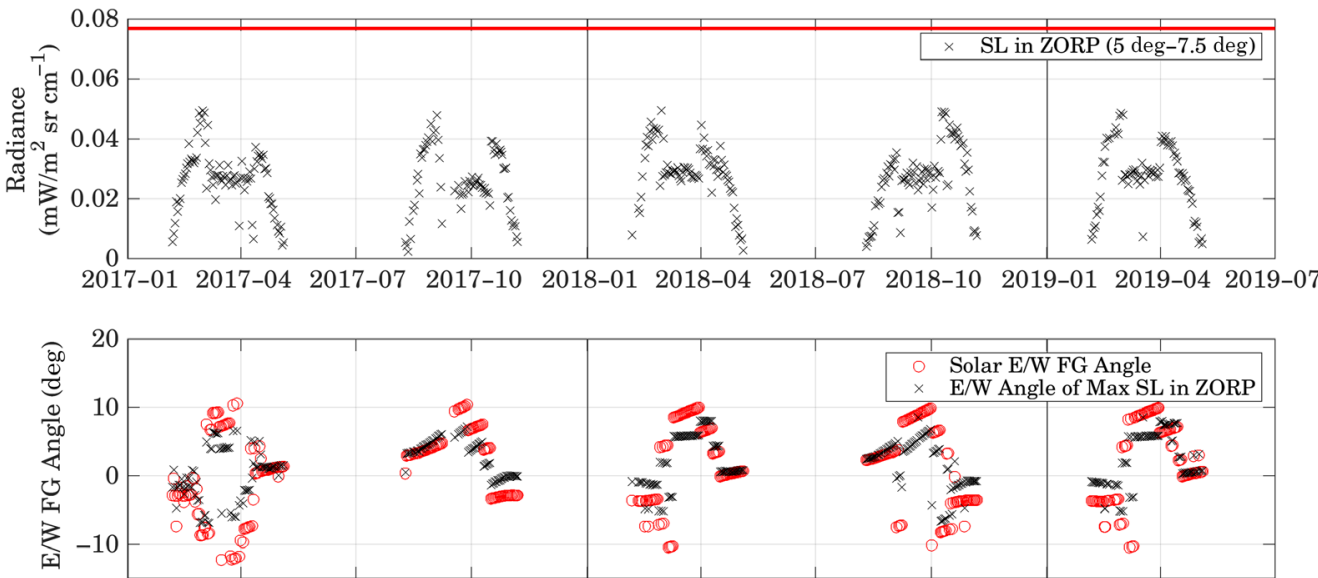

2017-01 2017-04 2017-07 2017-10 2018-01 2018-04 2018-07 2018-10 2019-01 2019-04 2019-07

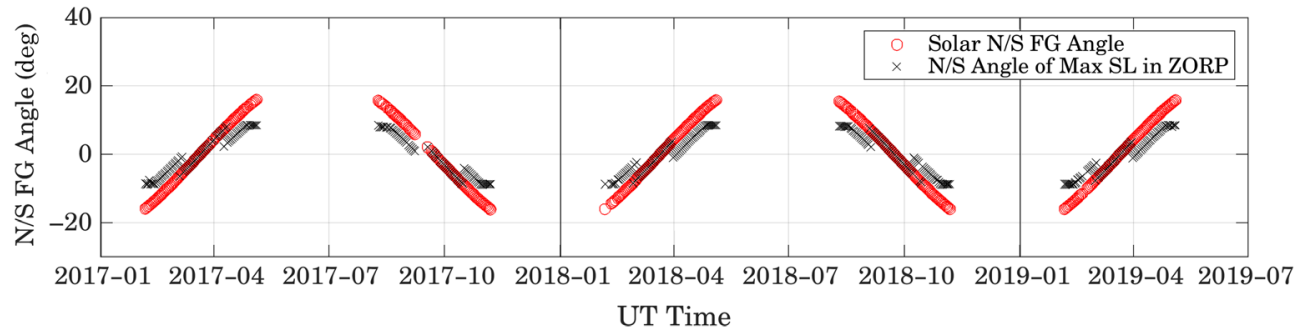

(a)
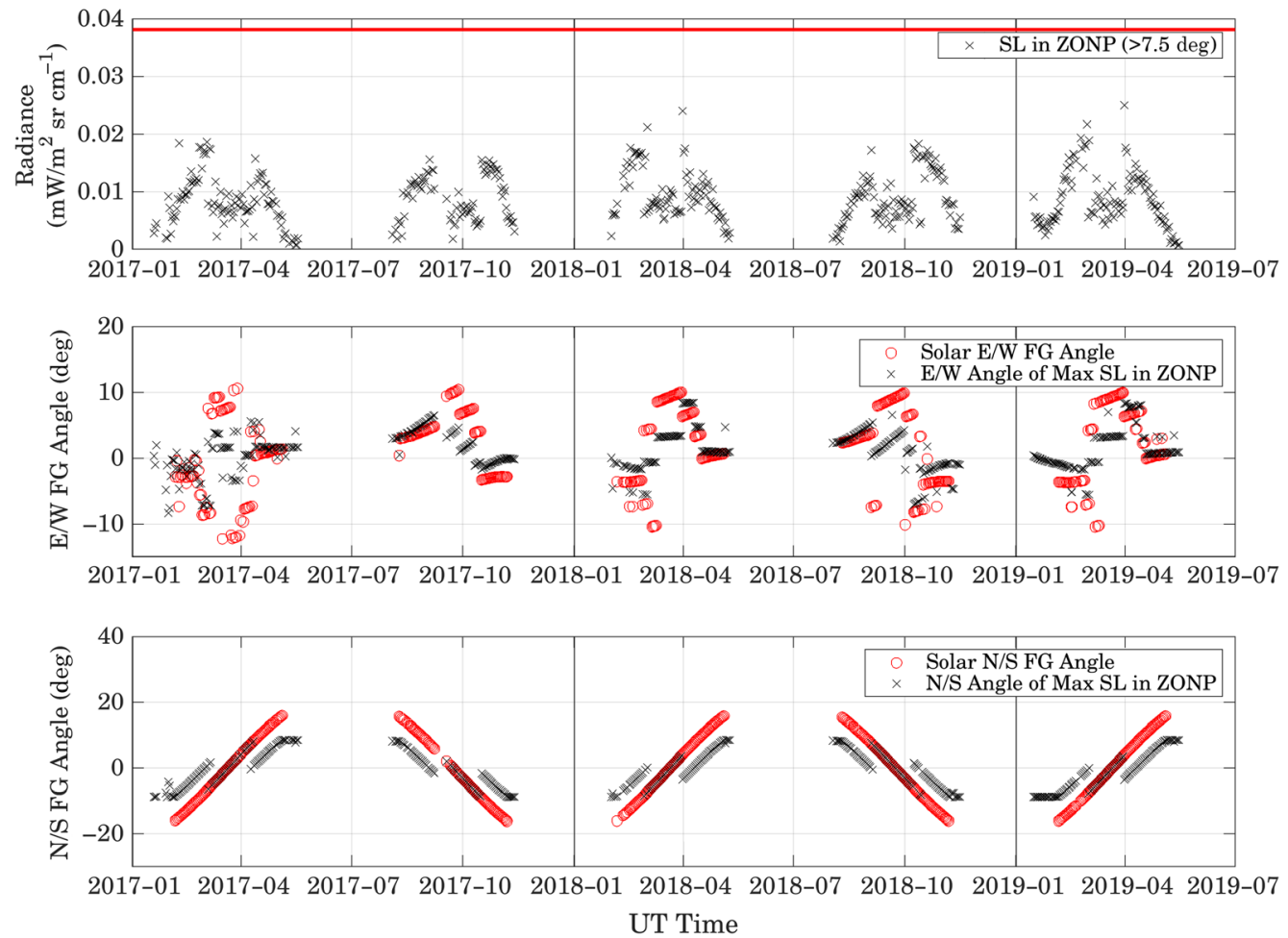

(b)

Fig. 8 Top panels in (a) and (b) show the long term trend of daily maximum stray-light magnitude inside ZORP and ZONP, respectively. The red line in each panel marks the radiometric requirement in the corresponding zone. Middle and bottom panels in (a) and (b) show E/W and N/S angular location of maximum stray light in ZORP and ZONP and the corresponding solar position in ABI-FGC, respectively. 
Table 4 GOES-16 ABI CH07 radiometric requirements and equivalent values of observed maximum stray light in radiance and brightness temperature as monitored in ZORP and ZONP.

\begin{tabular}{lll}
\hline \hline ABI CH07 & Radiometric requirement & \multicolumn{1}{c}{$\begin{array}{c}\text { Observed maximum } \\
\text { stray-light magnitude }\end{array}$} \\
\hline ZORP (5 deg $\left.<R_{\text {pixel-Sun }} \leq 7.5 \mathrm{deg}\right)$ & $\begin{array}{l}<0.076 \mathrm{~mW} / \mathrm{m}^{2} \mathrm{srcm}^{-1} \text { or } \\
\text { equivalently }<2 \mathrm{~K} \text { at } \\
300 \mathrm{~K} \text { scene }\end{array}$ & $\begin{array}{l}\sim 0.050 \pm 0.005 \mathrm{~mW} / \mathrm{m}^{2} \mathrm{srcm}^{-1} \text { or } \\
\text { equivalently } \sim 1.31 \pm 0.13 \mathrm{~K} \text { at } \\
300 \mathrm{~K} \text { scene }\end{array}$ \\
& $\begin{array}{l}<0.038 \mathrm{~mW} / \mathrm{m}^{2} \mathrm{srcm}^{-1} \text { or } \\
\text { equivalently }<1 \mathrm{~K} \mathrm{at}\end{array}$ & $\begin{array}{l}\sim 0.025 \pm 0.005 \mathrm{~mW} / \mathrm{m}^{2} \mathrm{srcm}^{-1} \text { or } \\
\text { equivalently } \sim 0.65 \pm 0.12 \mathrm{~K} \text { at } \\
\text { ZONP }\left(R_{\text {pixel-Sun }}>7.5 \mathrm{deg}\right)\end{array}$ \\
& $300 \mathrm{~K}$ scene & $300 \mathrm{~K}$ scene \\
\hline \hline
\end{tabular}

Earth during exclipse; and the final amplitude-decrease phase until the disappearnce of stray light as Sun exits the $~ 17$ - deg zone around Earth's equator. When the Sun is at the E/W side of Earth, the incident angle to $\mathrm{ABI}$ is more along the $\mathrm{E} / \mathrm{W}$ direction. In this case, there are stray light appearing in ABI FD image only when the Sun is close to the edge of Earth, and Earth can shield part of the ring around the solar disk. When the Sun is at the N/S side of Earth, the incident angle is along the N/S direction and stray light appear in midnight ABI FD images before the ring around the Sun is partially covered by Earth. This is why the stray light magnitude is lower when Sun is at the E/W side of Earth in comparison with the peak stray-light magnitude when the Sun is at $\mathrm{N} / \mathrm{S}$ side of Earth.

In Fig. 8, the N/S position of peak stray light in ZORP and ZONP closely follows the N/S movement of the solar position in ABI-FGC. The E/W angular position of the daily peak stray light mostly follows the solar position when Sun the is at the southern or northern side of Earth. When the Sun is near Earth's equator, the position of the daily peak stray light is several degree inward into the FD image relative to the solar position. It can be seen in Fig. 8, the ABI CH07 stray light almost repeats its appearance each time when Sun-ABI-Earth move to the same geometric configuration. This directly suggests that the stray light is related to the leaking of solar light into the ABI instrument.

The red horizontal lines in the top panels in Figs. 8(a) and 8(b) also show the radiometric uncertainty requirement in ZORP and ZONP, respectively. Table 4 summarizes the monitored maximum stray-light magnitude in radiance $\left(\mathrm{mW} / \mathrm{m}^{2} \mathrm{srcm}^{-1}\right)$ and equivalent temperature at $300 \mathrm{~K}$ scene in ZORP and ZONP for GOES-16 ABI CH07. The radiometric uncertainties for ABI CH07 need to be within $1 \mathrm{~K}$ and $2 \mathrm{~K}$ at equivalent $300 \mathrm{~K}$ scene in ZONP and ZORP, respectively. The maximum stray-light magnitude for GOES-16 ABI is $\sim 1.31 \pm 0.13 \mathrm{~K}$ at $300 \mathrm{~K}$ scene inside the ZORP and $\sim 0.65 \pm 0.12 \mathrm{~K}$ at $300 \mathrm{~K}$ scene inside ZONP, respectively. The uncertainty is derived from the residual of the exponential fitting of the averaged radiancedifference data as a function of $R_{\text {pixel-Sun }}$ angle. The stray-light contamination inside ZORP and ZONP are $\sim 35 \%$ below the required radiometric bias threshold for ABI CH07. Therefore, the long term stray-light monitoring shows that the radiometric performance of GOES-16 ABI CH07 is within the specification.

\subsection{Comparison of Stray Light in GOES-16 ABI CHO7 with Other Geostationary Imagers}

\subsubsection{Comparison of $\mathrm{CHO7}$ stray light between Himawari-8 AHI and GOES-16 ABI}

As mentioned in Sec. 1, the AHI instrument onboard Himawari-8 geostationary satellite was built by the same vendor and has very similar optical design as GOES-16 ABI. The AHI on Himawari-8 was launched on October 7, 2014, about 2 years before GOES-16 ABI. ${ }^{11-19}$ Intensive analysis of post-launch Himawari-8 AHI data provided early opportunity to evaluate the performance of this next-generation geostationary imaging sensor and prepare for ABI. One important lesson learned from AHI was that significant stray light was reported in VNIR channel and infrared $\mathrm{CH} 07$ and $\mathrm{CH} 08$ of $\mathrm{AHI} .{ }^{18,19}$ Characterization, monitoring, and trending of the stray 


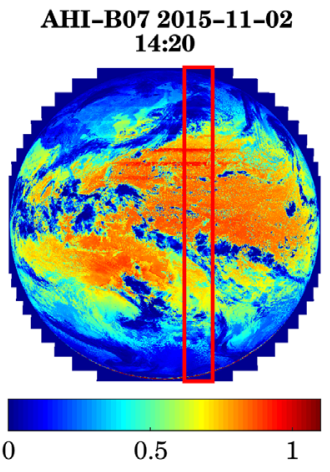

(a)

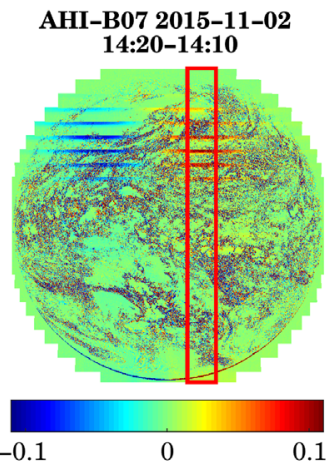

(b)

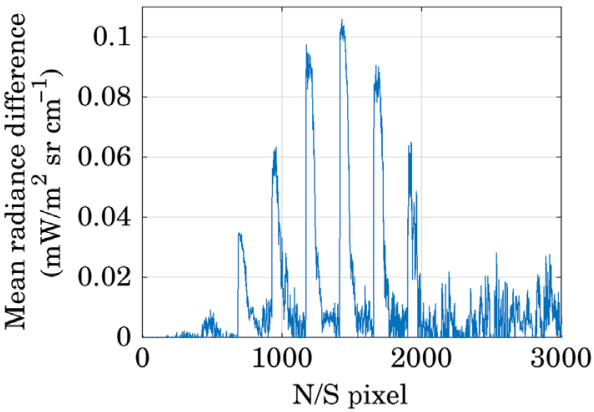

(c)

Fig. 9 (a) and (b) Radiance $\left(\mathrm{mW} / \mathrm{m}^{2} \mathrm{srcm}^{-1}\right)$ and time-difference radiance FD images derived from Himawari-8 AHI CH07 observations on November 2, 2015, respectively. The red rectangle illustrates the pixel region for profiling stray-light distribution along the N/S direction. (c) The mean radiance difference $\left(\mathrm{mW} / \mathrm{m}^{2} \mathrm{sr} \mathrm{cm}^{-1}\right)$ versus $\mathrm{N} / \mathrm{S}$ pixels. The mean value is calculated by averaging pixel values between pixel \#3100 and \#3600 along the E/W direction, i.e., within the red rectangle shown in (a) and (b). Data used for (b) and (c) are calculated from consecutive AHI CH07 observations at UT 14:10 and 14:20 on November 2, 2015.

light in AHI imagery product helped diagnose the origin of the stray light and assisted the instrument vendor to develop design fixes to remedy the stray light on GOES-16 ABI.

In this section, we characterize the stray light of Himawari-8 AHI CH07 and compare it to that of GOES-16 ABI CH07. Similar to GOES-16 ABI, the stray light in AHI CH07 FD imagery also appears over 3 month around spring and fall equinox. These stray-light moves with the solar position along both the N/S and E/W directions. But the position and magnitude of CH07 stray light in AHI is different from ABI.

Figure 9(a) shows example of the AHI CH07 FD imagery in radiance $\left(\mathrm{mW} / \mathrm{m}^{2} \mathrm{sr} \mathrm{cm}^{-1}\right)$ on November 2, 2015. There are faint horizontal eastward-moving stray-light stripes that appear as an overlay onto the upper part of the FD imagery. To show the stray light more clearly, Fig. 9(b) shows the time-difference radiance image derived from consecutive 10-min apart AHI observations. In Fig. 9(b), the horizontal blueish stripes in the northern part of the image are due to stray light in the previous AHI radiance image which has been subtracted. The yellow-reddish stripes to the east are due to stray light in the more recent AHI radiance image. There are $22 \mathrm{E} / \mathrm{W}$ swaths in total distributed along the N/S direction in each AHI FD image. As shown in Fig. 9(b), the horizontal stripes of stray light are clearly aligned within AHI swaths along the E/W direction and move from west-to-east following the Sun in the AHI-FGC.

The time-difference data shown in Fig. 9(b) were further processed to quantitatively assess the magnitude of AHI CH07 stray light. A region with clear stray light has been identified in Fig. 9(b) and marked with a red rectangle. The region spans between pixel \#3100 and \#3600 along the E/W direction. Figure 9(c) shows the mean radiance difference versus N/S pixels as derived from averaging pixel values in the red rectangle region along the $\mathrm{E} / \mathrm{W}$ direction. The AHI CH07 stray light shown in Fig. 9(c) has clear saw-tooth pattern with six spikes along the N/S direction. Each of these stray-light spikes is located within its corresponding AHI swath and in ZONP. The largest stray light spike is located around the N/S pixel \#1500 with amplitude as high as $0.104 \mathrm{~mW} / \mathrm{m}^{2} \mathrm{srcm}^{-1}$. The amplitude of adjacent stray-light spikes decreases as moving away from the swath with the largest stray-light spike. The mean Earth scene radiance in this region has large variation from $<0.148 \mathrm{~mW} / \mathrm{m}^{2} \mathrm{sr} \mathrm{cm}^{-1}$ over cloud to $\sim 0.816 \mathrm{~mW} / \mathrm{m}^{2} \mathrm{sr} \mathrm{cm}^{-1}$ over cloud-free Earth scene. The stray-light spikes in Fig. 9(c) are independent of scene radiance value. This further suggests that the stray-light is additive to the Earth scene signal observed by the AHI detector.

In comparison, the horizontally banded stray light in AHI image as shown in Fig. 9(b) are not identifiable in ABI image [Fig. 2(c)]. For ABI CH07, spike stray light (partially inside the ZORP) near the edge of FD image is the dominant source of stray light. Table 5 summarizes the maximum CH07 stray-light magnitude of Himawari-8 AHI and GOES-16 ABI. For Himawari-8 AHI, the maximum $\mathrm{CH} 07$ stray light occurred in ZONP and its magnitude can 
Table 5 Comparison of maximum $\mathrm{CH} 07$ stray-light magnitude among Himawari-8 $\mathrm{AHI}$, and GOES-16 and $-17 \mathrm{ABI}$.

\begin{tabular}{lccc}
\hline \hline & $\begin{array}{c}\text { Maximum stray light } \\
\text { (at 300 K) }(\mathrm{K})\end{array}$ & $\begin{array}{c}\text { Appearance } \\
\text { zone }\end{array}$ & $\begin{array}{c}\text { Corresponding radiometric } \\
\text { requirement (at 300 K scene) (K) }\end{array}$ \\
\hline Himawari-8 AHI & 3.35 & ZONP & 1 \\
GOES-16 ABI & 0.65 & ZONP & 1 \\
GOES-16 ABI & 1.31 & ZORP & 2 \\
GOES-17 ABI & 0.45 & ZONP & 1 \\
GOES-17 ABI & 1.07 & ZORP & 2 \\
\hline \hline
\end{tabular}

be as high as $\sim 0.133 \mathrm{~mW} / \mathrm{m}^{2} \mathrm{srcm}^{-1}$ or equivalently $\sim 3.35 \mathrm{~K}$ at $300 \mathrm{~K}$ scene, which is above the specification of $1 \mathrm{~K}$ at $300 \mathrm{~K}$ scene for this region. This comparison indicates that the design fix by vendor for reducing ABI sensor stray light is quite effective in removing the horizontally banded stray light. The remnant spike stray light at the edge of GOES-16 ABI CH07 FD image is below the radiometric requirements in both ZORP and ZONP as shown in Table 4. In conclusion, this analysis confirms that the overall $\mathrm{CH} 07$ stray-light contamination of GOES-16 ABI is less than that of Himawari-8 AHI. It also confirms the removal of strong stray-light leaking path for $\mathrm{ABI}$ after the design modifications by the instrument vendor as a result of lessons learned from Himawari-8 AHI.

There were identifiable stray light in both AHI CH07 and CH08 that can help to determine the cause of the stray light. Possible causes of stray light can be due to the leaking of solar light into the instrument after multiple reflections and then being detected by the AHI CH07 detectors; or due to the thermal emission from the heated thermal body onboard AHI after being illuminated by the solar light. Since the MWIR spectrum of the solar light and heat body emission are quite different, we show here the evidence of direct solar-origin of the stray light using the bandratio of AHI stray-light radiance in IR channels.

Figure 10(a) shows the stray light of AHI CH07 and CH08 which was derived similarly as those in Fig. 9(c). The AHI CH07 and CH08 are both MWIR channels with center wavelengths at $3.9 \mu \mathrm{m}$ and $6.2 \mu \mathrm{m}$, respectively. The stray light in AHI CH08 has similar spatial distribution along the N/S direction as that of the AHI CH07 and the amplitude is much smaller. Figure 10(b) further shows scatter plot of AHI stray light of $\mathrm{CH} 07$ versus $\mathrm{CH} 08$. The ratio of the stray-light

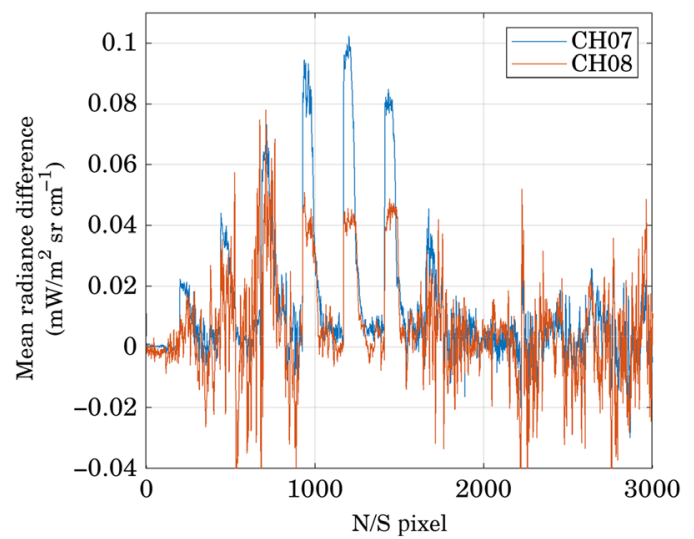

(a)

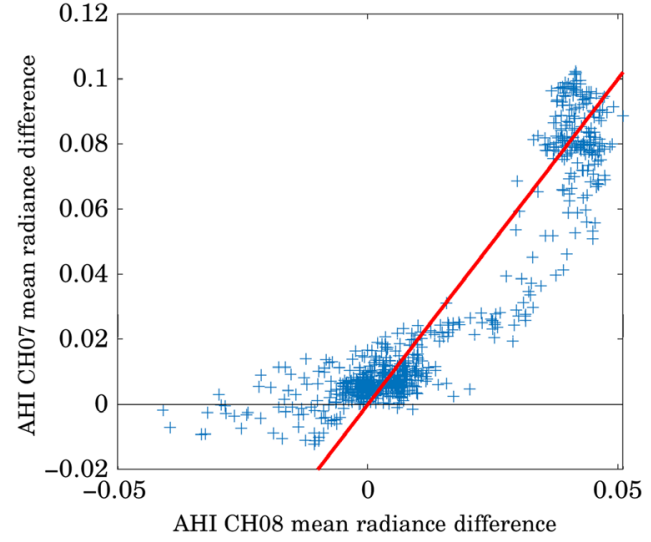

(b)

Fig. 10 (a) Mean time-difference radiance $\left(\mathrm{mW} / \mathrm{m}^{2} \mathrm{sr} \mathrm{cm}^{-1}\right)$ data for $\mathrm{AHI} \mathrm{CH} 07$ and $\mathrm{CH} 08$ versus $\mathrm{N} / \mathrm{S}$ pixel. The mean time-difference data are derived over the stray-light-contaminated region as indicated by the red rectangle in Fig. 9(b). (b) Scatter plot of AHI stray light $\left(\mathrm{mW} / \mathrm{m}^{2} \mathrm{sr} \mathrm{cm}^{-1}\right)$ of $\mathrm{CH} 07$ versus $\mathrm{CH} 08$ together with the linear regression line in red. Data between N/S pixel \#820 and \#1570 in (a) have been used to generate data points in (b). 
Table 6 Band-ratios of the maximum Himawari-8 AHI stray-light magnitude, solar radiance at Earth, and $300 \mathrm{~K}$ heat body radiation at $\mathrm{AHI} \mathrm{CH} 07$ and $\mathrm{CH} 08$ wavelengths.

\begin{tabular}{lcccc}
\hline \hline & $\begin{array}{c}\text { Center } \\
\text { wavelength } \\
(\mu \mathrm{m})\end{array}$ & $\begin{array}{c}\text { Maximum stray-light } \\
\text { magnitude } \\
\left(\mathrm{mW} / \mathrm{m}^{2} \mathrm{srcm}^{-1}\right)\end{array}$ & $\begin{array}{c}\text { Solar radiance } \\
\text { at earth } \\
\left(\mathrm{mW} / \mathrm{m}^{2} \mathrm{srcm}^{-1}\right)\end{array}$ & $\begin{array}{c}300 \mathrm{~K} \text { heat } \\
\text { body radiation } \\
\left(\mathrm{mW} / \mathrm{m}^{2} \mathrm{srcm}^{-1}\right)\end{array}$ \\
\hline $\mathrm{AHI} \mathrm{CH07}$ & 3.9 & 0.101 & 4.84 & 0.87 \\
$\mathrm{AHI} \mathrm{CH08}$ & 6.2 & 0.048 & 2.15 & 22.3 \\
Ratio $(\mathrm{CH} 07 / \mathrm{CH} 08)$ & - & 2.10 & 2.25 & 0.04 \\
\hline \hline
\end{tabular}

magnitude is $\sim 2$ between these two bands as derived from a linear fit. In Table 6 , the ratios of maximum AHI stray light, solar radiance at Earth with Sun's temperature $=5778 \mathrm{~K}$, and emission from $300 \mathrm{~K}$ heat body between $\mathrm{AHI} \mathrm{CH07}$ and $\mathrm{CH} 08$ are listed. The ratio of maximum stray light magnitude ( 2.1) between AHI CH07 and CH08 is consistent with the ratio of solar radiance $(\sim 2.25)$ for those two bands. This suggests that the AHI stray light in IR channels are due to the direct illumination of attenuated solar radiation onto the AHI detectors rather than the illumination from onboard thermal body emission.

\subsubsection{Comparison of GOES-16 and GOES-17ABI CHO7 stray light}

GOES-17 was launched about one-and-half-year after GOES-16. Both ABIs have the same design except that there was malfunction of loop heat pipe (LHP) on GOES-17 ABI after it was launched. ${ }^{8-10}$ The LHP transfers heat from the ABI electronics to the radiator and could not operate at its designed capacity on GOES-17 ABI. ${ }^{8,9}$ This caused difficulty in maintaining at the intended temperature for some of the ABI IR channel detectors during parts of night, leading to partial loss of ABI imagery. Details on GOES-17 ABI IR channel anomaly, mitigation, and radiometric performance can be found in Refs. 8-10 Similar to GOES-16 ABI, there were stray light observed in GOES-17 ABI CH07 imagery data during 3 months around spring and fall equinox. Using the stray-light characterization method outlined in Sec. 2.2, the stray light of GOES-17ABI CH07 was monitored as shown in Fig. 11. During October 28 to November 13, 2018 interval, GOES-17 drifted from $-89.5^{\circ}$ to $-137.2^{\circ} \mathrm{W}$ longitude to become GOES-West and

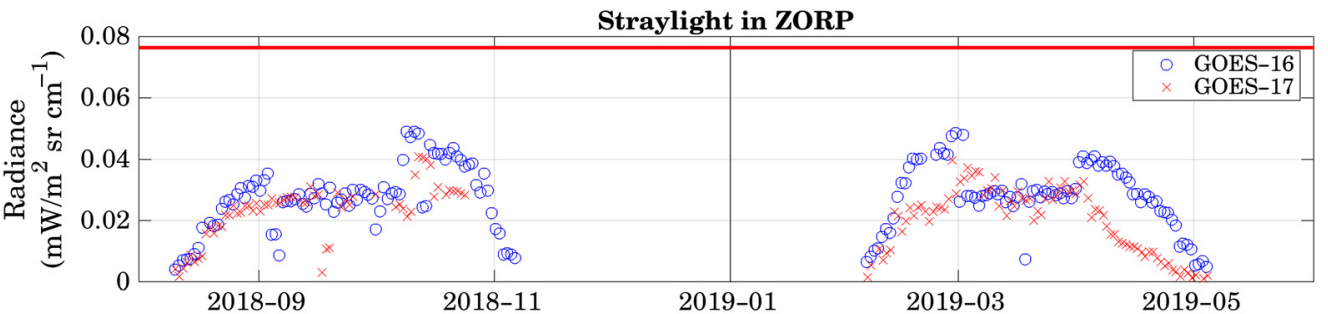

(a)

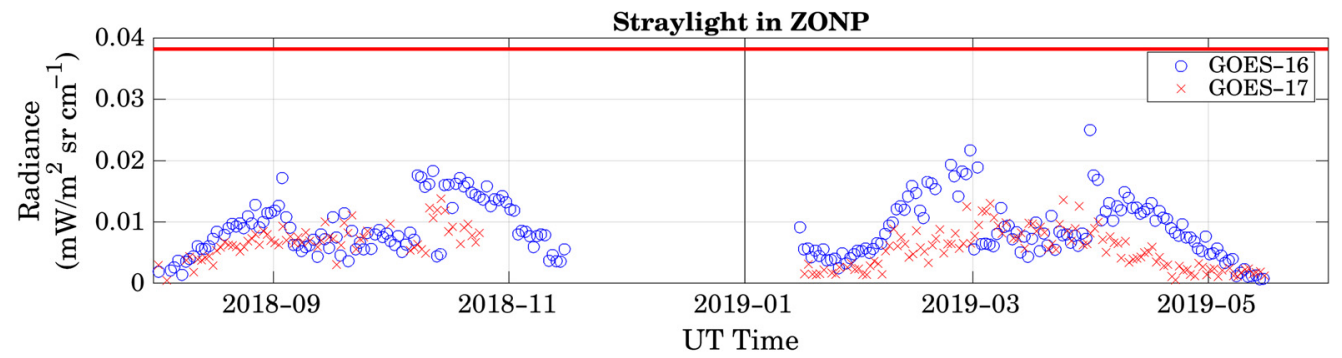

(b)

Fig. 11 Comparison of long term monitoring of GOES-16 and GOES-17 ABI CH07 stray light in (a) ZORP and (b) ZONP. The red line in each panel marks the radiometric requirement in the corresponding zone. 
Table 7 Equivalent maximum BT (K) error at different scene temperatures due to additive straylight radiance onto pixels in ZORP and ZONP for GOES-16 and GOES-17 ABI.

\begin{tabular}{lccccc}
\hline \hline & \multicolumn{2}{c}{$\begin{array}{c}\text { BT }(\mathrm{K}) \text { error for GOES-16 } \\
\text { ABI CH07 }\end{array}$} & & \multicolumn{2}{c}{$\begin{array}{c}\text { BT }(\mathrm{K}) \text { error for GOES-17 } \\
\text { ABI CH07 }\end{array}$} \\
\cline { 2 - 3 } Scene temperature $(\mathrm{K})$ & ZORP $(\mathrm{K})$ & ZONP $(\mathrm{K})$ & & ZORP $(\mathrm{K})$ & ZONP (K) \\
\hline 220 & 25.8 & 17.1 & 23.1 & 13.4 \\
240 & 12.9 & 7.4 & 11.0 & 5.40 \\
260 & 5.82 & 3.05 & 4.84 & 2.15 \\
280 & 2.66 & 1.34 & 2.19 & 0.93 \\
300 & 1.31 & 0.65 & 1.07 & 0.45 \\
320 & 0.70 & 0.34 & 0.57 & 0.24 \\
\hline \hline
\end{tabular}

no nighttime data were available. The maximum stray-light magnitude for GOES-17 ABI is $\sim 1.07 \pm 0.12 \mathrm{~K}$ at $300 \mathrm{~K}$ scene inside the ZORP and $\sim 0.45 \pm 0.12 \mathrm{~K}$ at $300 \mathrm{~K}$ scene inside ZONP. The GOES-17 ABI CH07 stray light is slightly lower than that of GOES-16 as summarized in Table 5. Therefore, the long term stray-light monitoring shows that the radiometric performance of GOES-17 ABI CH07 is also within the specification.

It is worth mentioning that although the stray-light magnitudes are within the radiometric requirements at $300 \mathrm{~K}$ scene for $\mathrm{ABI} \mathrm{CH} 07$ of both GOES-16 and GOES-17, the stray-light superimposed onto low temperature scenes such as cloud can cause substantially large brightness temperature (BT) error as shown in Table 7. The BT error of GOES-16 ABI CH07 can be as large as $25.8 \mathrm{~K}$ and $17.1 \mathrm{~K}$ in ZORP and ZONP, respectively, for a $220 \mathrm{~K}$ scene. Similarly, the BT error of GOES-17 ABI can be as large as $23.1 \mathrm{~K}$ and $13.4 \mathrm{~K}$ in ZORP and ZONP, respectively, for a $220 \mathrm{~K}$ scene. The users of GOES-16 and GOES-17 ABI L1B data should pay attention to such large BT error caused by the stray light during February-May and August-November season each year when analyzing ABI CH07 L1B data.

\section{GOES-16 ABI VNIR Channel Stray-Light Performance}

For GOES-16 ABI VNIR channels (CH01-CH06), substantial stray light around midnight were observed. There are no radiometric requirements on ABI VNIR channel data products around midnight. Therefore, the nighttime stray light in ABI VNIR channel data products do not violate any radiometric requirements. However, long-term monitoring of GOES-16 ABI VNIR channel stray light are routinely performed to help monitor instrument health and understand the root cause of ABI instrument stray light.

Figure 12 shows typical pattern of ABI VNIR channel stray light in radiance. Since the background of ABI VNIR channel FD image around midnight is dark, the yellow upward spike stray light at the southern part of the disk can be clearly identified without the need of calculating timedifference data. The location of the VNIR channel stray light co-register with that of ABI CH07. Figures 12(a) and 12(b) also show that the spike stray light moved from west-to-east in FD image along with Sun. The ABI CH06 has additional stray light in the upper part of the FD images (marked with a red box) which can be due to the different location of ABI CH06 detectors on the focal plane array. Striping in ABI CH02 nighttime image can also be seen. Since these stray lights in ABI VNIR channels only affect the imagery data around midnight, they are of less concern in comparison with the impacts of stray light on the ABI CH07 images.

To monitor the stray-light variation, ABI VNIR channel radiance data were used directly in stray-light analysis. The remaining steps of analyzing ABI VINR radiance data for characterizing stray light such as coordinate transformation and estimating stray-light magnitude are the same as those outlined in Sec. 2.2. For ABI VINR channel, the ZORP is defined as the interception region between the ABI FD image and the $3 \mathrm{deg}$ to $7.5 \mathrm{deg}$ zone around the Sun. The ZONP is defined as the region in ABI imagery with $R_{\text {pixel-Sun }}>7.5 \mathrm{deg}$. 


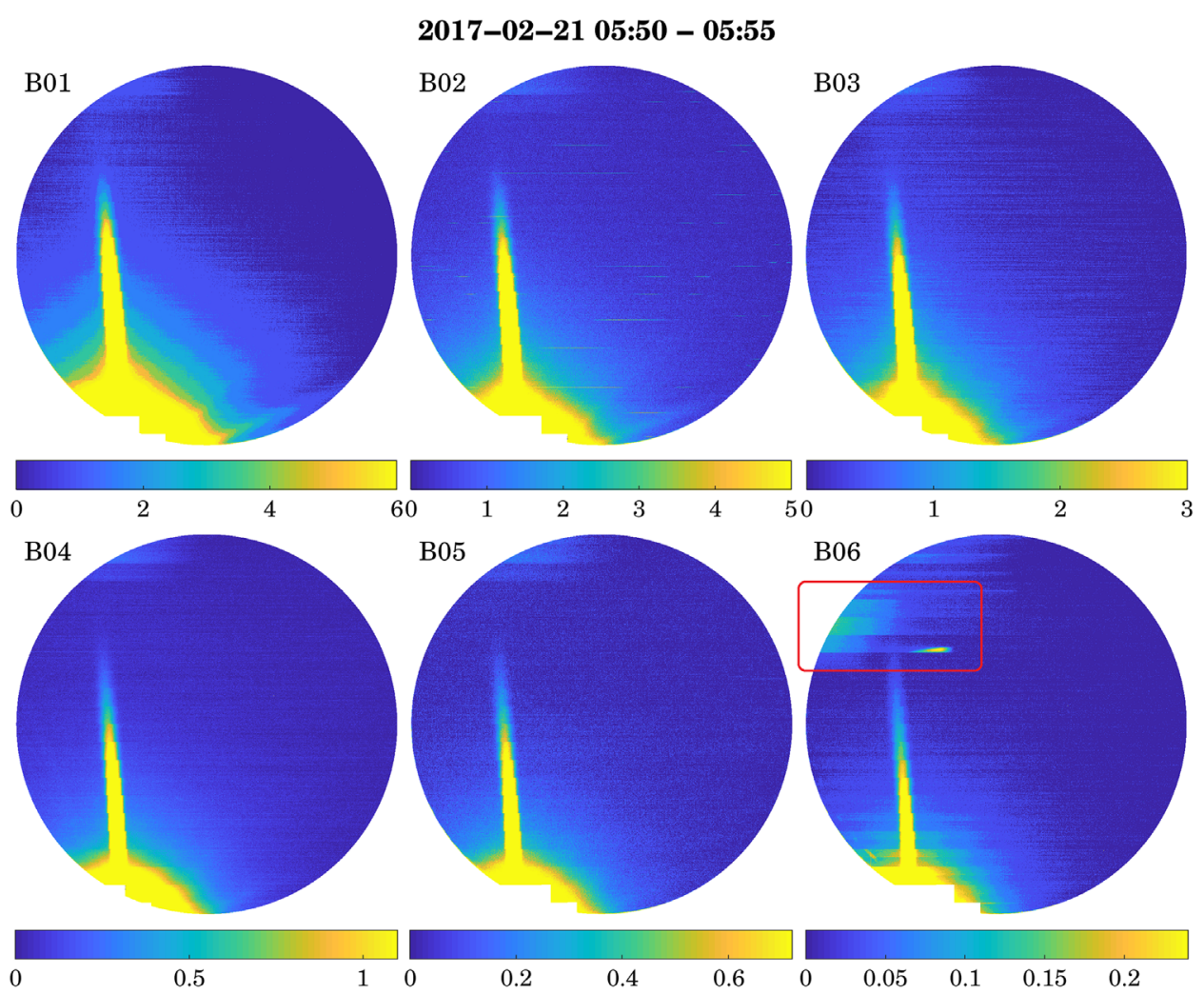

(a)

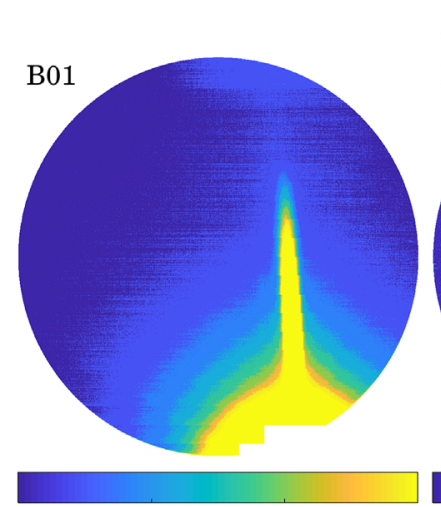

2017-02-21 06:20 - 06:25
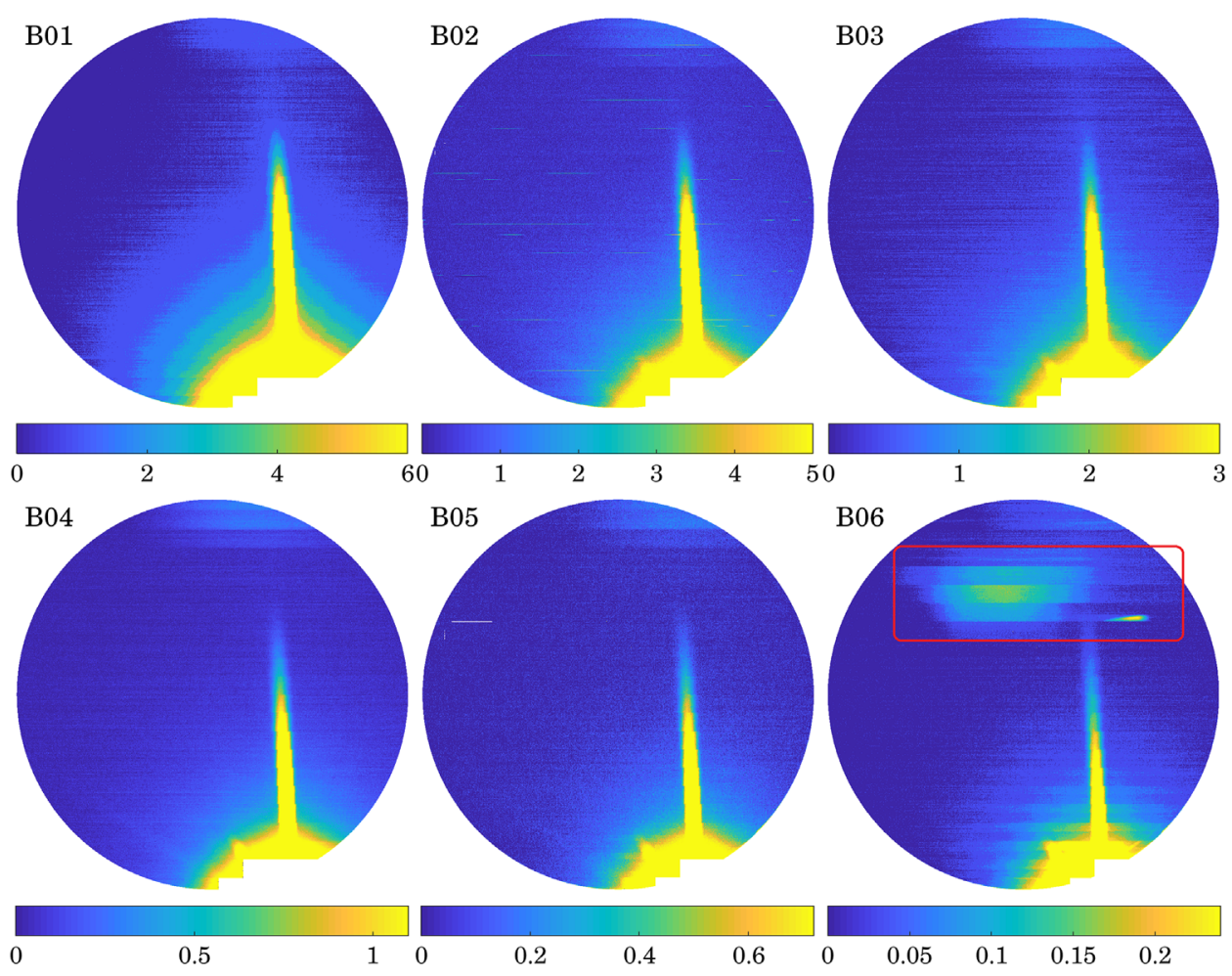

(b)

Fig. 12 Typical stray-light pattern in FD radiance $\left(\mathrm{W} / \mathrm{m}^{2} \mathrm{sr} \mu \mathrm{m}\right)$ image data for ABI VNIR CH01 to CH06 at two instants (a) 05:50 to 05:55 and (b) 06:20 to 0.6:25 around local midnight on February 21, 2017. The ABI FD data were collected under Mode 4. 

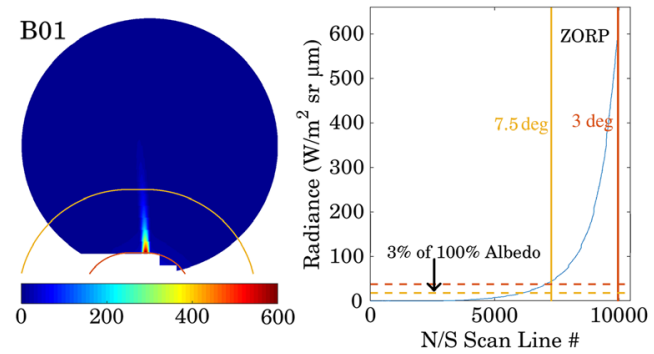

(a)
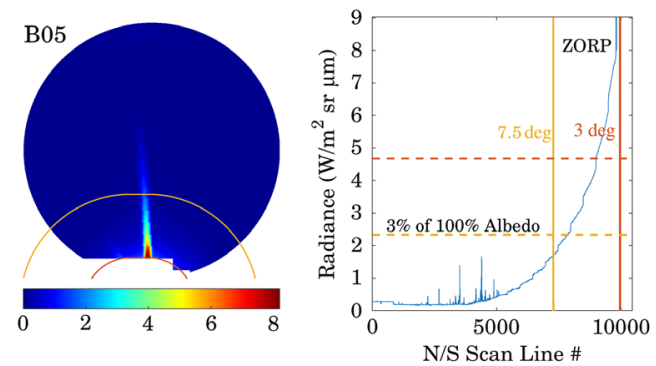

(c)

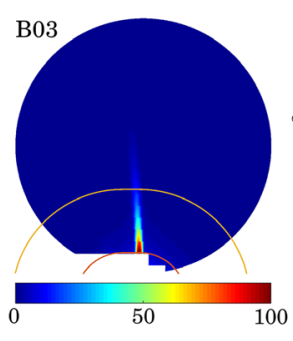

(b)
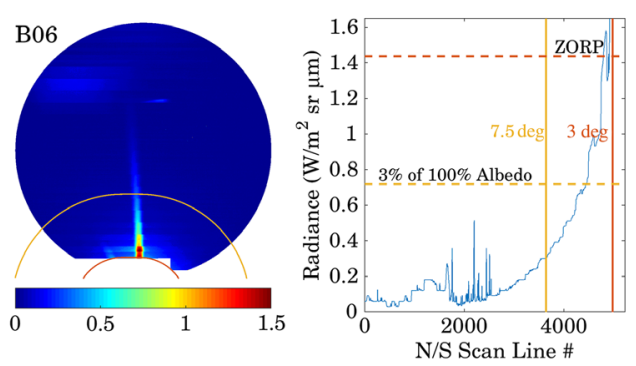

(d)

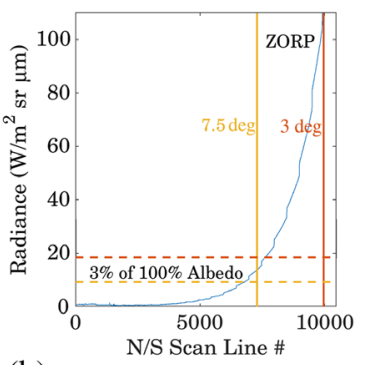
Fig. 13 (a)-(d) (left) Typical stray-light pattern in $\mathrm{ABI} \mathrm{CH} 01, \mathrm{CH} 03, \mathrm{CH} 05$, and $\mathrm{CH} 06$ radiance and $7.5 \mathrm{deg}$ (yellow line) around the Sun]. The color scale unit is $\mathrm{W} / \mathrm{m}^{2} \mathrm{sr} \mu \mathrm{m}$. (Right) Stray-light magnitude versus $A B I$ scan line number along the N/S direction. The 3 deg (vertical red line) and 7.5 deg (vertical yellow line) ZORP/ZONP boundaries, 6\% (red horizontal line), and 3\% (yellow horizontal line) of $100 \% \mathrm{ABI}$ albedo radiance are marked in the plot.

Typical stray-light pattern and stray-light magnitude versus ABI N/S scan line number for ABI CH01, CH03, CH05, and CH06 are shown in Fig. 13. The stray light of ABI VNIR channels have their maximums at the edge of the exclusion zone $\left(R_{\text {pixel-Sun }}=3 \mathrm{deg}\right)$ and decrease rapidly into the ABI FD along the N/S direction. In Figs. 13(c) and 13(d), there are additional stray light in the N/S scan line number region from 3000 to 4500 and from 1500 to 2250 for ABI CH05 and $\mathrm{CH} 06$, respectively. Since ABI CH05 has the pixel resolution twice of ABI CH06, the additional stray light affects the same N/S region on the CH05 and CH06 FD imagery.

Long-term monitoring of stray-light amplitude in ZORP and ZONP for GOES-16 ABI $\mathrm{CH} 01, \mathrm{Ch} 03, \mathrm{CH} 05$, and $\mathrm{CHO6}$ are shown in Fig. 14. The long-term variation of stray light is similar to ABI CH07 with ramping-up to reach peak when the Sun approaches Earth in N/S direction, being flatten in amplitude during eclipse season, and ramping-down when the Sun is away from the Earth. The maximum stray-light amplitude of ABI VINR channels occur when the Sun is at the southern or northern edge of Earth. The maximum stray-light magnitude inside and outside ZORP and ZONP for GOES-16 ABI VNIR channels (CH01-CH06) are given in Table 8. The spectral scaling of maxumum stray-light magnitude in ZORP matches the solar irrardaicne spectrum of ABI VNIR channels, which indicates the solar origin of the stray light in $\mathrm{ABI}$.

\section{$4 \mathrm{ABI}$ Solar Avoidance and Atmospheric Refraction-induced Stray-Light Contamination}

To avoid direct solar light contamination onto the ABI detectors and maintain healthy performance of ABI sensor, the solar avoidance was scheduled by truncating part of the ABI swaths according to angle between ABI pixel and solar position, i.e., $R_{\text {pixel-Sun}}$. These truncated partial swath data were not collected during solar avoidance by steering the ABI scan mirrors away from observing Earth scene. Figure 15 shows the GOES-16 ABI VNIR channel FD imagery with swath truncations for solar avoidance at UT 4:30 on April 2, 2018. Since there are E/W offsets among detector array for different ABI channels, the swath truncations for ABI VNIR channels 
Shao et al.: Characterization and monitoring of GOES-16 ABI stray light and comparison...

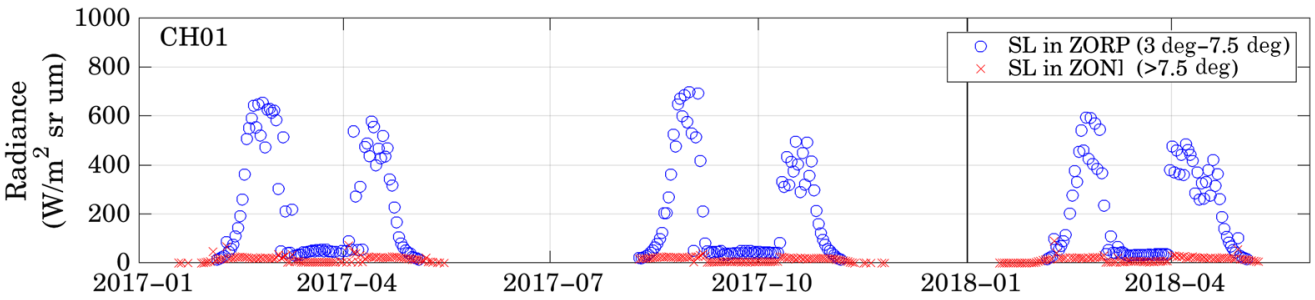

(a)

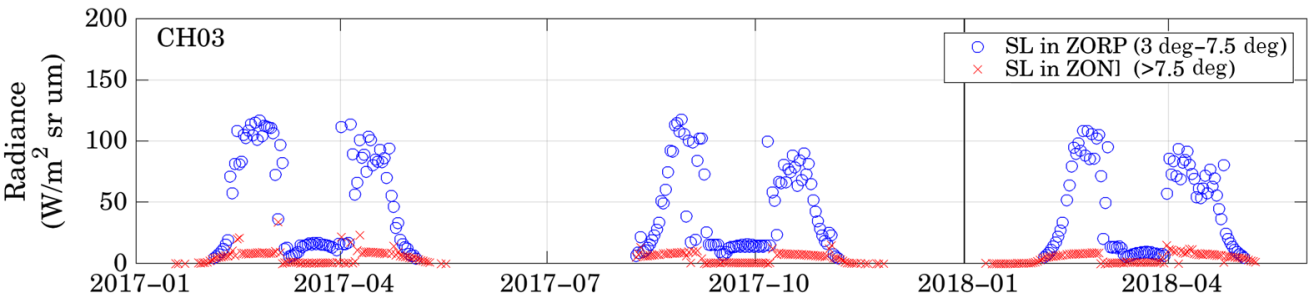

(b)

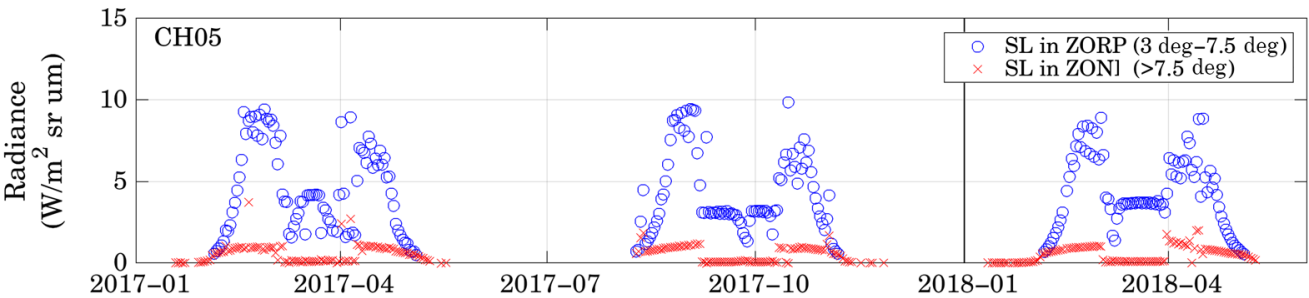

(c)

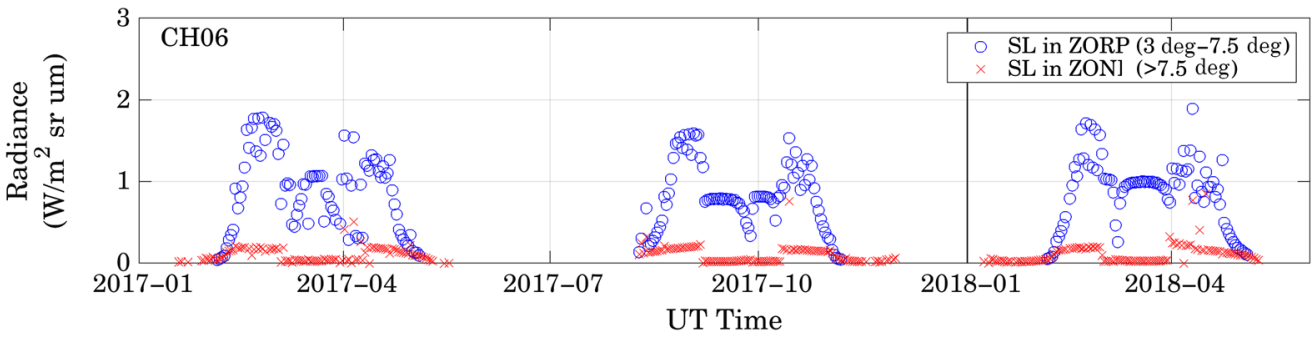

(d)

Fig. 14 (a)-(d) Long-term monitoring of maximum stray-light magnitude in radiance $\left(\mathrm{W} / \mathrm{m}^{2} \mathrm{sr} \mu \mathrm{m}\right)$ in ZORP (blue O) and ZONP (red+) for GOES-16 ABI CH01, $\mathrm{CH} 03, \mathrm{CH} 05$, and $\mathrm{CH} 06$, respectively.

Table 8 Summary of maximum stray-light inside ZORP and ZONP around local midnight for GOES-16 ABI VNIR channels $(\mathrm{CH01-CH06)}$ as derived from long-term monitoring.

\begin{tabular}{lcccccc}
\hline \hline & $\mathrm{CH} 01$ & $\mathrm{CH} 2$ & $\mathrm{CH} 3$ & $\mathrm{CH} 0$ & $\mathrm{CH} 0$ & $\mathrm{CH} 06$ \\
\hline Wavelength $(\mu \mathrm{m})$ & 0.47 & 0.64 & 0.86 & 1.37 & 1.6 & 2.2 \\
Inside ZORP $\left(\mathrm{W} / \mathrm{m}^{2} \mathrm{sr} \mu \mathrm{m}\right)$ & 692.3 & 372.5 & 126.9 & 18.1 & 9.96 & 1.86 \\
Inside ZONP $\left(\mathrm{W} / \mathrm{m}^{2} \mathrm{sr} \mu \mathrm{m}\right)$ & 76.2 & 46.7 & 46.1 & 2.8 & 3.80 & 0.90 \\
\hline \hline
\end{tabular}

appear to have corresponding E/W shifts in ABI-FGC. Comparing ABI CH04 and CH06 which are at the opposite far end of the $\mathrm{ABI}$ focal plan array, we can see that there are clear shifts in truncation locations. In particular, ABI CH04 observes more stray light due to being closer the west side. The solar avoidance can also cause some channels to have missing data in pixels closer to the eclipse regions around Sun, which have direct impact on the assembled RGB color imagery products in those regions. 

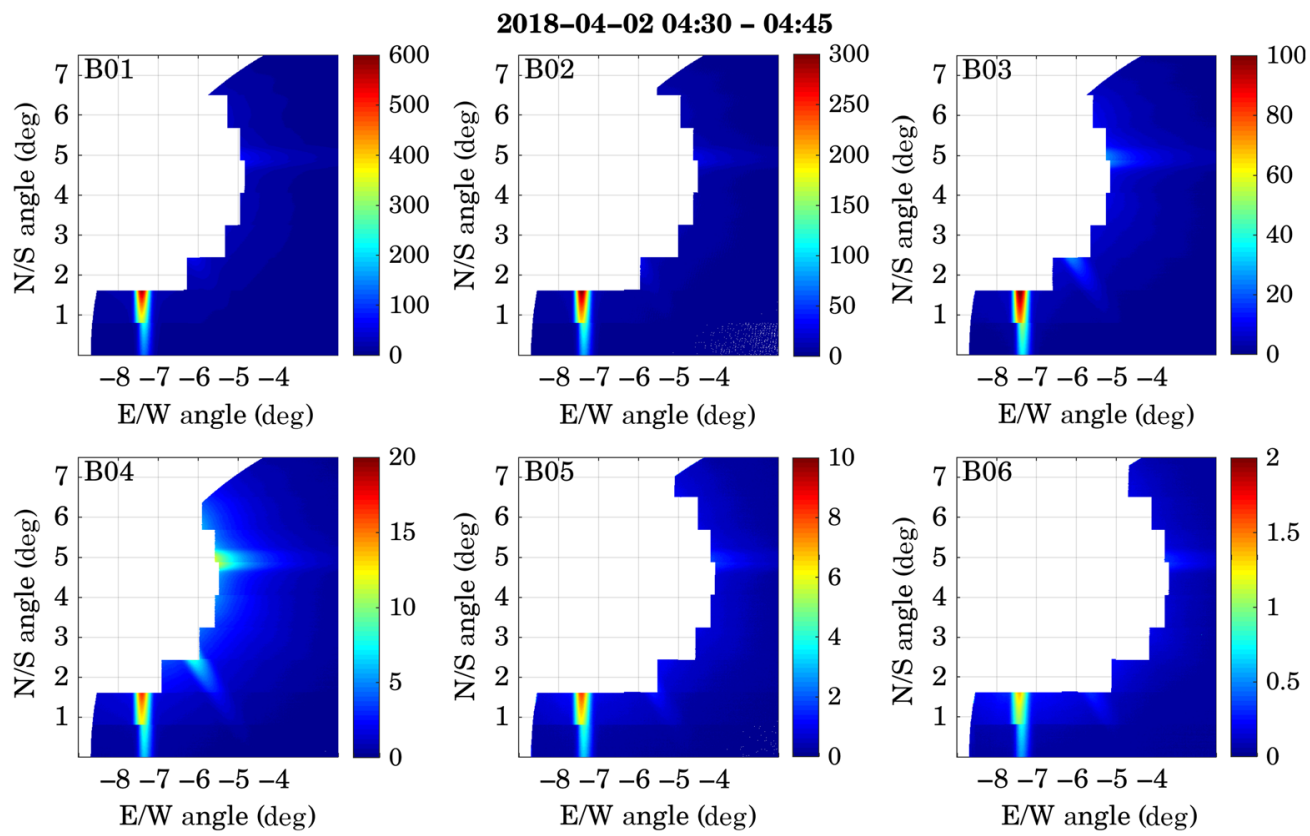

Fig. 15 Example of solar avoidance-swath truncation for GOES-16 ABI VNIR channels. The radiance unit of the color bar in each panel is $\mathrm{W} / \mathrm{m}^{2} \mathrm{srum}$.

The view-geometry parameter of GOES-16 ABI solar avoidance was initially set with an annular circle with radius $R_{\text {limb }}=8.1 \mathrm{deg}$, i.e., with limb margin $\sim 0.5 \mathrm{deg}$ inside the Earth's FD of $R_{\text {Earth }} \sim 8.6 \mathrm{deg}$. This inner annular ring was set to account for the $\sim 0.5 \mathrm{deg}$ solar disk diameter and atmospheric effects. When the solar center is inside $R_{\text {limb }}$, it was assumed that the direct solar light contamination onto the ABI sensor detectors could be avoided and further swath truncations were unnecessary. However, during the solar eclipse season, it was observed that there were strong stray-light irradiation onto the ABI sensor detectors even when the solar center is inside the $R_{\text {limb }}=8.1 \mathrm{deg}$ annular circle. Figures 16(a)-16(c) show example of strong stray-light contamination onto GOES-16 ABI CH03 imagery data during March 2018. The swath truncations are shown as white-filled rectangles and are calculated for each swath according to the solar position at the start and end of the swath. In these three cases [Figs. 16(a)-16(c)], when the solar position relative to Earth (portion of green line) was already inside the yellow circle $\left(R_{\text {limb }}=8.1 \mathrm{deg}\right)$, there were un-truncated swaths contaminated by solar light with radiance above $373.167 \mathrm{~W} / \mathrm{m}^{2}$ sr um which saturated the detector [see inside red oval in Figs. 16(a)-16(c)]. Exposure to these strong stray-light contamination are unhealthy for ABI detectors. It was found that the atmospheric refraction effects allow portions of Sun to be visible even when the Sun is geometrically located fully behind the earth. The initial solar avoidance setting with $R_{\text {limb }}=8.1$ degree for GOES-16 ABI did not account for the effect of atmospheric refraction.

The refraction angle of solar light through atmosphere can be as large as $\sim 0.6 \mathrm{deg}^{20}$ when the incident light is tangential to Earth's surface, i.e., with solar zenith angle approaching $90 \mathrm{deg}$. When the Sun is behind the Earth, the maximum bending angle is estimated to $\sim 1.2$ deg since the solar light can bend twice as going through atmosphere due to the reciprocal symmetry of light propagation. The solution to avoid these atmospheric refraction-induced stray-light contamination is to reduce $R_{\text {limb }}$ value and enforce additional swath truncations. Given the solar radius of $\sim 0.25 \mathrm{deg}$, the radius of inner annular circle can be set as $R_{\text {limb }}=7.15 \mathrm{deg}$ as a test for avoiding atmospheric refraction-induced stray-light contamination. Figures 16(d)-16(f) show examples of additional swath truncations for three ABI CH03 FD images collected during March, 2018 after using solar avoidance parameter $R_{\text {limb }}=7.15 \mathrm{deg}$. It can be seen that the additional swath truncations shown as hollow yellow rectangles in Figs. 16(d)-16(f) can help avoid all of the strong stray-light-contamination in these three cases. The actual value of $R_{\text {limb }}$ in operation was set by the ABI vendor and can be different from the value given above. 


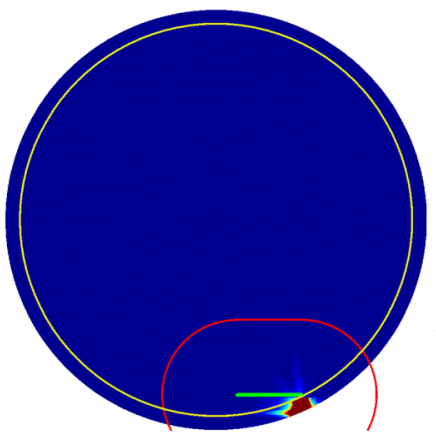

2018-03-02 $\left(R_{\text {limb }}=8.1 \mathrm{deg}\right)$

(a)

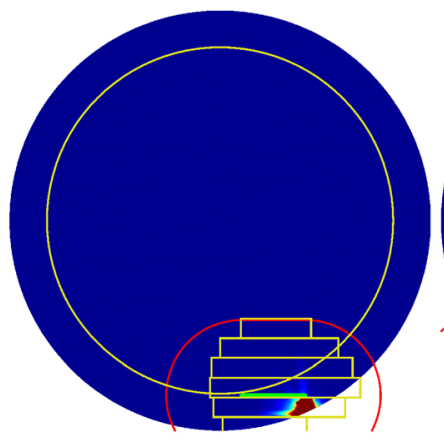

2018-03-02 $\left(R_{\text {limb }}=7.15 \mathrm{deg}\right)$

(d)

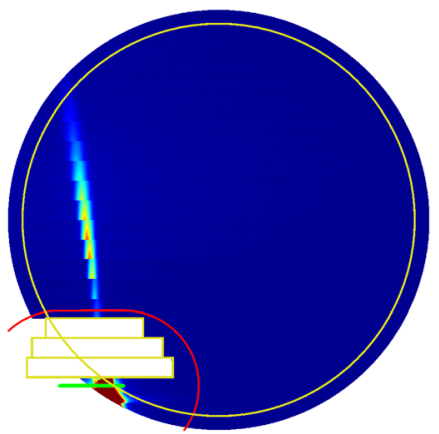

2018-03-03 $\left(R_{\text {limb }}=8.1 \mathrm{deg}\right)$

(b)

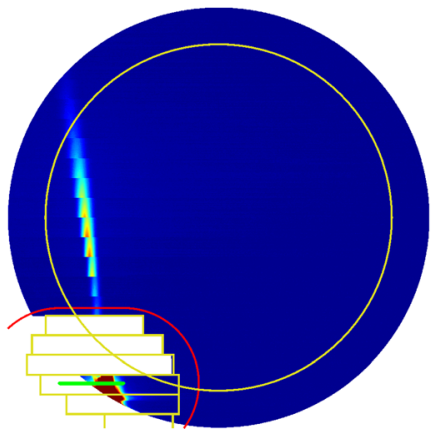

2018-03-03 $\left(R_{\text {limb }}=7.15 \mathrm{deg}\right)$

(e)

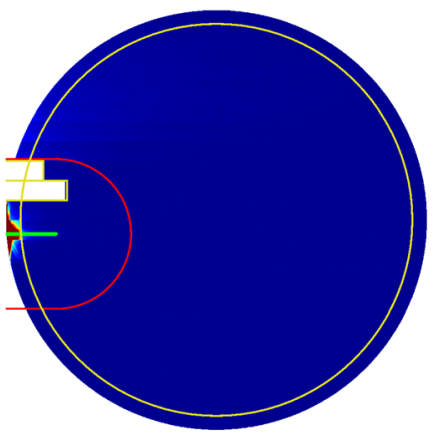

$2018-03-19\left(R_{\text {limb }}=8.1 \mathrm{deg}\right)$

(c)

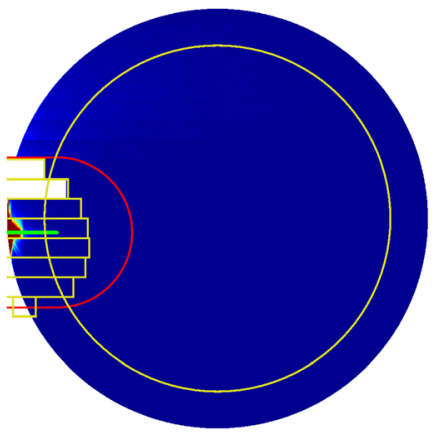

$2018-03-19\left(R_{\text {limb }}=7.15 \mathrm{deg}\right)$

(f)

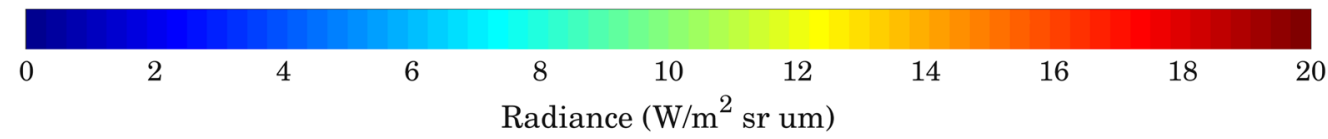

Fig. 16 (a)-(c) Examples of atmospheric refraction-induced strong stray-light contamination onto GOES-16 ABI CH03 imagery data during March 2018. Swath trunctions (white-filled yellow rectangles) are set up with $R_{\text {limb }}=8.1 \mathrm{deg}$ (yellow circle). (d)-(e) Demontration of additional swath trunctions (holow yellow rectangles) to avoid atmospheric refraction-induced strong straylight contamination by setting $R_{\text {limb }}=7.15 \mathrm{deg}$ (yellow circle). Green lines in each panel shows the movements of the Sun during the 15-min ABI Mode 3 FD image scan time. Red oval is the 3-deg exclusion zone moving along with the Sun. Yellow rectangles indicate the predicted swath truncations.

The $R_{\text {limb }}$ was reduced in operation in August 2018 and the effect can be seen in GOES-16 ABI data during the eclipse season in September 2018. With the implementation of reduced $R_{\text {limb }}$, the GOES-16 ABI detectors are better protected.

\section{Summary}

This paper developed a stray-light characterization scheme to quantitatively monitor the occurrence, location, and magnitude of stray-light variation in GOES-16 and -17 ABI and Himawari-8 AHI imagery data. The processing scheme consists of time-difference processing to remove the varying background IR emissions at night, SCC transformation to align stray light with solar origin, and estimation of stray-light magnitude with exponential fitting of averaged timedifference data in FD imagery.

The stray-light analysis is at first focused on GOES-16 ABI CH07 since the impacts of stray light on the nighttime radiometric performance of this channel are of major concern. It is found that over the 3 month interval around spring and fall equinox each year, the stray light in both ZONP and ZORP of ABI have similar time-variation pattern with different amplitudes. The diurnal appearance of stray light occurs within about $1.5 \mathrm{~h}$ around the local midnight at 
ABI nadir. The maximum stray-light magnitude for GOES-16 ABI CH07 is $\sim 1.31 \mathrm{~K}$ and $\sim 0.65 \mathrm{~K}$ at $300 \mathrm{~K}$ scene inside ZORP and ZONP, respectively, which are both $\sim 35 \%$ below the required radiometric bias thresholds for ABI CH07. The long term stray-light monitoring of GOES-17 ABI CH07 shows that its stray light are also within the radiometric specification and are slightly lower than those of GOES-16 ABI. It is noted that due to the additive nature of stray light, the errors in BT over low temperature scene such as cloud due to stray-light contamination can be large. For example, the BT errors of both GOES-16 and -17 ABI CH07 can be $>20 \mathrm{~K}$ and $>10 \mathrm{~K}$ in ZORP and ZONP, respectively, over a $220 \mathrm{~K}$ cold scene. The users of $\mathrm{ABI}$ and $\mathrm{AHI} \mathrm{CH} 07$ radiance data should be aware of the impacts of stray light on the low temperature scene during the February to May and August to November eclipse season each year.

The GOES-16 ABI CH07 stray-light pattern and magnitude are also compared with Himawari-8 AHI. For ABI CH07, spike stray light (partially inside the ZORP) near the edge of FD image is the dominant source of stray light. It is shown that the maximum stray light of Himawari-8 AHI CH07 appears as the horizontally banded stray light and its magnitude is $\sim 3.35 \mathrm{~K}$ at $300 \mathrm{~K}$ scene, which is much higher than that of GOES-16 ABI CH07. This paper confirms that the remediation fixes applied to GOES-16 and -17 ABI by the vendor for reducing the leaking paths of strong horizontally banded stray light were quite successful.

We also found that the stray light is the largest in AHI $\mathrm{CH} 07$ but also is detectable in other AHI MWIR channels. Analysis of the ratio of stray light magnitudes between AHI $3.9 \mu \mathrm{m}$ and $6.2 \mu \mathrm{m}$ band indicates that it is consistent with the ratio of solar radiance for these two bands. This suggests that the stray light is mainly due to direct illumination of the attenuated solar radiation onto the AHI detectors instead of from onboard thermal body emission due to heating.

Characterization and long term monitoring of GOES-16 ABI VNIR channel stray light are also performed to help understand the solar origin of ABI stray light. The long term stray-light variation pattern of ABI VNIR channels is similar to that of ABI CH07. The maximum stray light of ABI VINR channels occur when Sun is at the southern or northern edge of Earth. The ABI CH06 has additional stray light in the upper part of the FD image, which can be due to the edge location of ABI CH06 detectors on the focal plane array. The stray light in ABI VNIR channels only affect the imagery data around midnight and are of less concern in comparison to those of ABI CH07. Observations of Moon and stars for the calibration of ABI or AHI VNIR channels during the stray-light occurrence configuration, i.e., around midnight over spring and autumn equinox season, should be planned carefully since the stray-light might contaminate the observations.

This paper also investigated cases of strong atmospheric refraction-induced stray-light contamination onto GOES-16 ABI detectors during solar eclipse season when Sun is geometrically located fully behind the earth. Such exposure to strong stray light contamination are harmful for ABI detectors. We found that the initial solar avoidance setting for GOES-16 ABI did not account for the effect of atmospheric refraction. This paper demonstrates the scheme to avoid or reduce such potentially harmful strong stray-light contamination by changing ABI solar avoidance parameter setting. The refraction of solar light by atmospheric limb and its avoidance as shown in this paper should also be applicable to AHI and other geostationary imagers since the nature of solar light refraction through atmosphere only depends on the geometric configuration among Sun, Earth, and geostationary satellite.

The long term characterization and monitoring of ABI stray light also suggests the repeatability of both temporal variation and spatial distribution of ABI stray light. By organizing ABI stray light in SCC, it is feasible to prepare time-dependent stray light correction table for ABI using Artificial Intelligence (AI)-type classification and learning algorithms. There have been time-dependent stray-light correction in operation such as the monthly stray-light correction table prepared for SNPP and NOAA-20 Day Night Band stray-light correction. ${ }^{21,22}$ We suggest that the stray correction for $\mathrm{ABI} \mathrm{CH} 07$ can be explored in future research.

The presence of stray light in geostationary imaging sensor data is always a challenge. The analysis method for stray-light characterization presented in this paper can also be applied to other geostationary imaging sensors such as those operated by Korea Meteorological Administration (KMA) and The European Organisation for the Exploitation of Meteorological Satellites (EUMETSAT). 


\section{Acknowledgments}

This study was supported by NOAA GOES-R program through grant NA19NES4320002 (Cooperative Institute for Satellite Earth System Studies -CISESS) at the University of Maryland/ESSIC. Part of the work would not be possible without the AHI data through an agreement between NOAA and JMA to cooperate on respective advanced geostationary meteorological satellites, and numerous constructive discussions with JMA colleagues including Arata Okuyama, Masaya Takahashi, Takura Miyakawa, Katao Bessho, and Toshiyuki Kurino. We also thank the helpful discussions with John Van Naarden and Daniel Gall of Harris Corporation. The manuscript contents are solely the opinions of the authors and do not constitute a statement of policy, decision, or position on behalf of NOAA or the U.S. government. Disclosures: The authors have no relevant financial interests in the manuscript and no other potential conflicts of interest to disclose.

\section{References}

1. R. Datla et al., "Comparison of the calibration algorithms and SI traceability of MODIS, VIIRS, GOES, and GOES-R ABI sensors," Remote Sens. 8, 126 (2016).

2. T. J. Schmit et al., "A closer look at the ABI on the GOES-R series," Bulletin of the American Meteorological Society 98, 681-698 (2017).

3. F. Yu et al., "Early radiometric calibration performances of GOES-16 Advanced Baseline Imager," Proc. SPIE 10402, 104020S (2017).

4. F. Yu et al., "Validation of early GOES-16 ABI on-orbit geometrical calibration accuracy using SNO method," Proc. SPIE 10402, 104020U (2017).

5. S. Kalluri et al., "From photons to pixels: processing data from the Advanced Baseline Imager," Remote Sens. 10(2), 177 (2018).

6. X. Jing et al., "Validation of GOES-16 ABI VNIR channel radiometric performance with NPP and NOAA-20 VIIRS over the Sonoran Desert," J. Appl. Remote Sens. 14(4), 044517 (2020).

7. F. Yu et al., "Radiometric calibration accuracy of GOES-16 ABI infrared data," J. Appl. Remote Sens., to be submitted (2021).

8. J. V. Naarden and D. Lindsey, "Saving GOES-17,” 2019, https://aerospaceamerica.aiaa.org/ departments/saving-goes-17.

9. J. McCorkel et al., "GOES-17 Advanced Baseline Imager performance recovery summary," in IEEE Int. Geosci. Remote Sens. Symp., Yokohama (2019).

10. Z. Wang et al., "On-orbit calibration and characterization of GOES-17 ABI IR bands under dynamic thermal condition," J. Appl. Remote Sens. 14(3), 034527 (2020).

11. A. Okuyama et al., "Preliminary validation of Himawari-8/AHI navigation and calibration," Proc. SPIE 9607, 96072E (2015).

12. P. Griffith, "Advanced Himawari Imager (AHI) design and operational flexibility," in 12th Annu. Symp. New Gener. Oper. Environ. Satell. Syst., AMS Annu. Meeting (2016).

13. F. Yu and X. Wu, "Radiometric inter-calibration between Himawari-8 AHI and SNPP VIIRS for the solar reflective channels," Remote Sens. 8(3), 165 (2016).

14. K. Bessho et al., "An introduction to Himawari-8/9-Japan's new-generation geostationary meteorological satellites," J. Meteor. Soc. Jpn. 94, 151-183 (2016).

15. F. Yu et al., "Evaluation of Himawari-8 AHI geospatial calibration accuracy using SNPP VIIRS SNO data," in IEEE Int. Geosci. Remote Sens. Symp., Beijing, pp. 2925-2928 (2016).

16. X. Shao et al., "Comparison of Suomi-NPP VIIRS and HIMAWARI-8 AHI MWIR observations for hot spot and heat island studies," in IEEE Int. Geosci. Remote Sens. Symp., Beijing, pp. 1954-1957 (2016).

17. T. Tabata et al., "Himawari-8/AHI latest performance of navigation and calibration," Proc. SPIE 9881, 98812J (2016).

18. X. Shao, X. Wu, and F. Yu, "Characterization of Himawari-8 AHI 3.9-um channel stray light," Proc. SPIE 9972, 99720R (2016).

19. X. Shao, X. Wu, and F. Yu, "Stray-light performance comparison between Himawari-8 AHI and GOES-16 ABI," in IEEE Int. Geosci. Remote Sens. Symp., Fort Worth, Texas, pp. 286289 (2017). 
20. F. Kneizys et al., The MODTRAN 2/3 Report and LOWTRAN 7 MODEL, Phillips Laboratory, Hanscom AFB, Massachusetts (1996).

21. X. Shao et al., "Characterization and correction of stray light for NOAA-20 VIIRS day/night band," Proc. SPIE 10781, 107810H (2018).

22. X. Shao et al., "A light contamination ranking index-based method for automating VIIRS day/night band stray light correction," Proc. SPIE 10764, 107641I (2018).

Xi Shao received his BS degree in space physics from the University of Science and Technology of China in 1996, the PhD in astronomy in 2001, and an MS degree in electrical engineering with microelectronics major in 2004, both from the University of Maryland, College Park, Maryland. $\mathrm{He}$ is currently an associate research scientist at Cooperative Institute for Satellite Earth System Studies (CISESS), Earth System Science Interdisciplinary Center (ESSIC), University of Maryland.

Xiangqian Wu coleads the GOES-R Calibration Working Group (CWG) at NOAA in Maryland, USA, specializing in ABI calibration. He has been leading the operational calibration for NOAA's AVHRR on POES since 2002, Imager and Sounder on GOES since 2004, and OMPS on S-NPP between 2010 and 2014. He has been a member of the WMO-sponsored Global Space-based Inter-Calibration System (GSICS) Research Working Group since its inception, and served as its first chair.

Fangfang Yu is the lead of GOES-R ABI cal/val support team at the Cooperative Institute for Climate and Satellites, University of Maryland, supporting the operational calibration of NOAA's GOES imaging instruments. She has been supporting and working on the calibration and validation of NOAA's operational optical instruments since 2005. Her major research interest is focused on the radiometric calibration and validation of the geostationary weather satellite instruments.

Changyong Cao is a supervisory physical scientist with the NOAA Center for Satellite Applications and Research. He specializes in the calibration of radiometers onboard NOAA's Operational Environmental Satellites, and currently leads the VIIRS sensor science team. He was the recipient of three gold and one silver medals honored by the U.S. Department of Commerce for his scientific and professional achievements. He received his $\mathrm{PhD}$ from the Louisiana State University in 1992. 